\title{
Simultaneous near-field and far-field spatial quantum correlations in the high-gain regime of parametric down-conversion
}

\author{
E. Brambilla, A. Gatti, M. Bache, and L. A. Lugiato \\ INFM, Dipartimento di Scienze CC FF MM, Università dell'Insubria, Via Valleggio 11, 22100 Como, Italy
}

(Received 15 May 2003; published 9 February 2004)

\begin{abstract}
We study the spatial correlations of quantum fluctuations that can be observed in multimode parametric down-conversion in the regime of high gain. We investigate both a type-I and a type-II phase-matching configuration: in the latter case spatial correlations at the quantum level are shown to exist both in the near-field and in the far-field zones of the down-converted light. In the stationary and plane-wave approximation we treat the problem analytically. A stochastic model is solved numerically to obtain quantitative results beyond this approximation. The finite transverse size and pulse duration of the pump beam and other features of the system, such as spatial walk-off and diffraction are taken into account, and we show that correlations beyond the standard quantum limit exist for values of parameters consistent with realistic experiments.
\end{abstract}

DOI: 10.1103/PhysRevA.69.023802

PACS number(s): 42.50.Dv, 42.65.-k

\section{INTRODUCTION}

The spatial aspects of correlations of quantum optical fluctuations have been the object of several studies in the past [1-11]. In general they show up in nonlinear optical processes, typically wave-mixing phenomena which involve a large number of spatial modes of the electromagnetic field. From the middle of the 1990s there has been a renewal of attention because of new potential applications which exploit the quantum properties of the field for image processing or multichannel operations. Examples are quantum holography [12], the quantum teleportation of optical images [13], and the measurement of small displacements beyond the Rayleigh limit [14]. An overview of this relatively new branch of quantum optics, for which the name quantum imaging was coined, can be found in Ref. [15].

The process of frequency down-conversion is particularly suitable for this kind of application because of its large emission bandwidth in the spatial frequency domain. We consider parametric down-conversion (PDC) taking place in a crystal with a second-order nonlinearity set in a traveling-wave configuration. In this process the photons of a high-intensity pump field are split into pairs of photons of lower energy and momentum through the nonlinear interaction with the medium. Since no signal field is injected, down-conversion is initiated only by vacuum fluctuations that equally cover all spatial and temporal frequencies. The fluorescence pattern that arises has therefore the angular spectrum determined by phase-matching conditions, and depends only on the linear dispersion properties of the nonlinear material.

The spatial aspects of quantum correlations in PDC have been initially studied mainly in the low-gain regime. In this case, the mean number of photons per mode is small compared to unity and single-photon pairs can be resolved in time by the detectors. Information on spatial quantum correlations can therefore be obtained directly from coincidence measurements. A detailed theory has been developed in order to evaluate the two-photon coincidence rate using a perturbative method to determine the two-photon entangled state generated in the nonlinear process [3]. This theory has been applied to explain the outcomes of "two-photon entangled imaging" experiments. These exploit the entanglement of the two-photon state in order to retrieve information from an object inserted in the path of one of the photons by detecting the position of its twin [4]. Both geometrical and coherence aspects of the physics underlying these two-photon imaging experiments have been carefully examined [5-10].

In this paper we focus on spatial correlations in the opposite regime, where the number of photons per mode in the emitted field can be quite large, and their detection gives rise to continuous photocurrents. Under these conditions the perturbative approach used in Refs. [3,4] fails and an analytical expression of the output state can be formulated only within the stationary and plane-wave pump approximation (PWPA).

Recently, in the PWPA framework we used a multimode theory in order to demonstrate that PDC is able to display correlation effects in the spatial domain, both when photonnumber $[16,17]$ and polarization [18] measurements are considered. In these papers it is shown that the correlations of quantum origin, usually investigated in the photon-counting regime, survives in a regime of high gain where a large number of photons are emitted in each mode. In particular, the theory predicts noise reduction well below the shot-noise level for the difference in the number of photons measured in the far-field from two detection areas $R_{1}$ and $R_{2}$ corresponding to couples of phase-conjugate (signal and idler) modes. In other terms, the photon numbers measured over the two detection areas are identical even at the quantum level. This phenomenon finds its explanation at the microscopic level in the conservation of the photon transverse momentum which is fulfilled in each elementary down-conversion process: for each photon detected in, say, area $R_{1}$, the detection of its twin in $R_{2}$ is ensured by this law. The same kind of spatial correlations have also been extensively investigated in the continuous wave $(\mathrm{cw})$ pumped optical parametric oscillators, where the down-conversion efficiency is enhanced by enclosing the nonlinear crystal inside an optical resonator [11].

Here we shall give a description of the photon-number spatial correlations that can be observed in a traveling-wave configuration, both in type-I and in type-II phase-matching conditions. In the second case, we shall focus our attention 
on the spatial correlation property of PDC displayed in the near field, a problem that we never analyzed before. The signal and idler beams are found to exhibit quantum correlated photon-number fluctuations when measured from detection areas that image the same portion of the beam cross section. Twin photons are indeed generated simultaneously and they remain localized in a limited region of space as long as they are observed close to the crystal. This "position entanglement" of the generated photon pairs can be seen as the near-field counterpart of the momentum entanglement which can be observed in the far field. However, we shall see that for a realistic crystal length the measurement of near-field correlation is strongly affected by propagation effects, in particular diffraction and spatial walk-off. We shall propose a procedure to overcome at least partially this problem.

It should be noticed that the existence of correlations both in the near and in the far field of PDC is known in the literature in the coincidence counting regime [10]. It has been used in two-photon imaging experiments [4] and has been recently emphasized in a more general context [19]. In this work we focus on aspects that are specific of the highgain regime, because we study the spatial correlation functions of the photon number, determining the condition under which the signal-idler correlation beats the standard quantum limit.

The observation of photon-number correlation phenomena in the high-gain regime of PDC is also the aim of an experiment presently performed at the University of Insubria in Como. In this experiment photodetection takes place by means of a high quantum efficiency charged coupled device (CCD) camera, which is able to resolve photon number fluctuations that are below the standard quantum limit [20]. The pump field is a high-power picosecond laser pulse that provides energy for a large number of down-converted photons, in a configuration such that the plane-wave and $\mathrm{cw}$ pump approximations are very raw. Here we shall present a realistic description of the system, based on a numerical model that includes the finite duration and the transverse size of the pump pulse. Other features of the system that are relevant from an experimental point of view, such as spatial and temporal walk-off, different kinds of linear dispersion and phasematching (type-I and type-II crystals), are included in the model. It is important to investigate how they affect the spatial quantum correlation phenomena predicted by the planewave pump theory, also in order to identify the best conditions under which they can be observed in the experiment. A numerical evaluation of the far-field photon-number correlation function is presented in Ref. [21] in the case of a type-I crystal at degeneracy. In Ref. [21], which treats downconversion within a classical framework, the shape of the pump pulse included into the numerical model is taken from experimental data and the obtained signal-idler correlation peak displayed between symmetrical point reproduces well the correlation measured experimentally.

The paper is organized as follows. In Sec. II we briefly introduce the theoretical model used to describe PDC within a classical framework. The quantum description of the system is illustrated in Sec. III, where a fully analytical treatment is developed in the framework of the plane-wave and cw pump approximation. It is based on a multimode inputoutput formalism, first introduced in Ref. [22] for a type-I crystal at degeneracy, which is here extended to a type-II phase-matching configuration.

In Sec. IV we give a qualitative description of the phasematching mechanism that determines both the photonnumber distribution and the characteristic bandwidths of the down-converted field, illustrating thereby the differences between type-I and type-II phase matching.

In Sec. $\mathrm{V}$ we define the quantities that can be measured experimentally and that put in evidence the quantum nature of the spatial correlations in which we are interested. Their analytical expressions are derived within the PWPA, which will be used to interpret the results of the numerical model.

The last part of the paper (Sec. VI) is devoted to present the numerical results obtained for two particular crystals with different phase matching (type I and type II). The amount of correlation that may be achieved is evaluated as a function of different parameters that can be varied experimentally, such as the size of the pump beam waist and the size of the detectors.

\section{CLASSICAL DESCRIPTION OF THE PROCESS}

We decompose the electric field in the superposition of three quasimonochromatic wave packets (denoted with $E_{0}$, $E_{1}$, and $E_{2}$ ) of central frequencies $\omega_{0}, \omega_{1}$, and $\omega_{2}$, corresponding to the pump, signal, and idler fields, respectively. These frequencies are taken to satisfy the energyconservation condition $\omega_{1}+\omega_{2}=\omega_{0}$. Assuming the mean direction of propagation is the $z$ direction, and denoting with $\vec{x}=(x, y)$ the coordinate vector in the transverse plane, we can write

$$
E_{j}(z, \vec{x}, t) \propto A_{j}(z, \vec{x}, t) e^{i k_{j} z-i \omega_{j} t}+\text { c.c. } \quad(j=0,1,2),
$$

where $k_{j}=n_{j} \omega_{j} / c$ is the wave number of wave $j$ at the carrier frequency along the $z$ axis (for an extraordinary wave the refraction index $n_{j}$ depends on the propagation direction, a property leading to spatial walk-off). To simplify the notation we have ignored the vectorial character of the three fields, their polarization being determined by the kind of phasematching conditions that are met inside the crystal.

Within the paraxial and slowly varying envelope approximation, the propagation equations for the signal and idler (S-I) field envelopes and the pump field envelope can be written in the form [23]

$$
\begin{aligned}
\frac{\partial A_{j}}{\partial z} & +k_{j}^{\prime} \frac{\partial A_{j}}{\partial t}+\frac{i}{2} k_{j}^{\prime \prime} \frac{\partial^{2} A_{j}}{\partial t^{2}}-\rho_{j} \frac{\partial A_{j}}{\partial y}-\frac{i}{2 k_{j}} \nabla_{\perp}^{2} A_{j} \\
& =\sigma A_{0} A_{l}^{*} e^{-i \Delta_{0} z} \quad(j, l=1,2 ; j \neq l), \\
\frac{\partial A_{0}}{\partial z} & +k_{0}^{\prime} \frac{\partial A_{0}}{\partial t}+\frac{i}{2} k_{0}^{\prime \prime} \frac{\partial^{2} A_{0}}{\partial t^{2}}-\rho_{0} \frac{\partial A_{0}}{\partial y}-\frac{i}{2 k_{0}} \nabla_{\perp}^{2} A_{0} \\
& =-\sigma A_{1} A_{2} e^{i \Delta_{0} z}
\end{aligned}
$$


The driving terms on the right-hand side describe the wavemixing process due to the second-order nonlinearity of the medium, the coupling constant $\sigma$ being proportional to the effective second-order susceptibility $\chi_{\text {eff }}^{(2)}$ characterizing the down-conversion process. $\Delta_{0}=k_{1}+k_{2}-k_{0}$ is the collinear phase mismatch of the central frequency components.

Linear propagation is described by the lhs of these equations: the terms proportional to $k_{j}^{\prime}=\left(\partial k_{j} / \partial \omega\right)_{\omega=\omega_{j}}$ and $k_{j}^{\prime \prime}$ $=\left(\partial^{2} k_{j} / \partial \omega^{2}\right)_{\omega=\omega_{j}}$ lead to temporal walk-off between the different waves and group-velocity dispersion, respectively, while the terms containing the first- and second-order derivatives in the transverse coordinates $(x, y)$ are responsible for spatial walk-off and diffraction, respectively. $\rho_{j}$ indicates the walk-off angle of wave $j$, determined by the anisotropy of the crystal (the walk-off direction is taken along the $y$ axis). Linear losses are neglected, so that the three waves exchange energy but their total energy is conserved.

In Sec. VI A we shall also consider the special case of a type-I phase-matched crystal where the signal and the idler fields have the same polarization and are observed close to the degenerate frequency $\omega_{1}=\omega_{2}=\omega_{0} / 2$. Under these conditions the signal and idler fields are no more distinguishable and the down-converted field must be described by a single slowly-varying envelope $A(z, \vec{x}, t)$ satisfying the following propagation equation:

$$
\frac{\partial A}{\partial z}+k^{\prime} \frac{\partial A}{\partial t}+\frac{i}{2} k^{\prime \prime} \frac{\partial^{2} A}{\partial t^{2}}-\rho_{1} \frac{\partial A}{\partial y}-\frac{i}{2 k} \nabla_{\perp}^{2} A=\sigma A_{0} A^{*} e^{-i \Delta_{0} z},
$$

which is readily obtained from Eqs. (2a) by dropping the S-I indices $j, l$, which denote different polarizations and/or carrier frequencies in the nondegenerate case.

In a single-pass configuration with crystal length on the order of a few millimeters, the amplitudes of the downconverted field remain small with respect to the pump amplitude and the nonlinear driving term in the rhs of Eq. (2b) can be neglected. The pump depletion due to downconversion and absorption is indeed of small entity, unless extremely high intensity laser sources are used. We shall therefore work within the parametric approximation that treats the pump as a known classical field which propagates linearly inside the crystal, while the down-converted fields are quantized according to rules that are briefly illustrated in the following section.

\section{QUANTUM DESCRIPTION IN THE PARAMETRIC APPROXIMATION}

We need now to substitute the classical signal and idler fields with operators. Making the formal substitution for the field envelopes $A_{j}(z, \vec{x}, t) \rightarrow a_{j}(z, \vec{x}, t) \quad(j=1,2)$, we impose the following commutation rules at equal $z[1]$ :

$$
\begin{gathered}
{\left[a_{i}(z, \vec{x}, t), a_{j}^{\dagger}\left(z, \vec{x}^{\prime}, t^{\prime}\right)\right]=\delta_{i j} \delta\left(\vec{x}-\vec{x}^{\prime}\right) \delta\left(t-t^{\prime}\right),} \\
{\left[a_{i}(z, \vec{x}, t), a_{j}\left(z, \vec{x}^{\prime}, t^{\prime}\right)\right]=0 \quad(i, j=1,2),}
\end{gathered}
$$

valid within the framework of the paraxial and quasimonochromatic approximations. With this definition

$$
I_{j}(z, \vec{x}, t)=a_{j}^{\dagger}(z, \vec{x}, t) a_{j}(z, \vec{x}, t) \quad(j=1,2)
$$

is the photon flux density operator associated with wave $j$ : its expectation value gives the mean number of photons crossing a region of unit area in the transverse plane. In the linear regime the field operators obey the same equations as the corresponding classical quantities. For our purpose, it is useful to introduce the Fourier transforms of the field envelopes with respect to time and to the transverse plane coordinates:

$$
a_{j}(z, \vec{q}, \Omega)=\int \frac{d \vec{x}}{2 \pi} \int \frac{d t}{\sqrt{2 \pi}} a_{j}(z, \vec{x}, t) e^{-i \vec{q} \cdot \vec{x}+i \Omega t} \quad(j=1,2)
$$

A similar definition holds also for the Fourier component $A_{0}(z, \vec{q}, \Omega)$ of the classical pump field envelope. The propagation equations (2a) take then the form

$$
\begin{aligned}
& \frac{\partial a_{j}(z, \vec{q}, \Omega)}{\partial z} \\
& =i \delta_{j}(\vec{q}, \Omega) a_{j}(z, \vec{q}, \Omega)+\sigma e^{-i \Delta_{0} z} \int \frac{d \vec{q}^{\prime}}{2 \pi} \int \frac{d \Omega^{\prime}}{\sqrt{2 \pi}} \\
& \quad \times A_{0}\left(z, \vec{q}-\vec{q}^{\prime}, \Omega-\Omega^{\prime}\right) a_{l}^{\dagger}\left(z,-\vec{q}^{\prime},-\Omega^{\prime}\right) \\
& (j, l=1,2 ; j \neq l),
\end{aligned}
$$

where

$$
\delta_{j}(\vec{q}, \Omega)=k_{j}^{\prime} \Omega+\frac{1}{2} k_{j}^{\prime \prime} \Omega^{2}+\rho_{j} q_{y}-\frac{1}{2 k_{j}}\left(q_{x}^{2}+q_{y}^{2}\right) \quad(j=1,2),
$$

is the quadratic expansion of $k_{j z}\left(\omega_{j}+\Omega, \vec{q}\right)-k_{j}$ around $\vec{q}$ $=\overrightarrow{0}, \Omega=0$, and $k_{j z}\left(\omega_{j}+\Omega, \vec{q}\right)=\sqrt{k_{j}^{2}\left(\omega_{j}+\Omega, \vec{q}\right)-q^{2}}$ denotes the $z$ component of the $k$ vector associated with the $(\vec{q}, \Omega)_{j}$ plane-wave mode. In particular the walk-off angle $\rho_{j}$ can be identified as $\partial k_{j} / \partial q_{y}$ calculated for $\vec{q}=0, \Omega=0$. A more detailed derivation can be found in $[1,24]$.

Equations (7) contain the convolution integral in Fourier space of the S-I field envelope with the pump field envelope. Within the undepleted pump approximation, the latter can be expressed as

$$
\begin{gathered}
A_{0}(z, \vec{q}, \Omega)=e^{i \delta_{0}(\vec{q}, \Omega) z} A_{0}(z=0, \vec{q}, \Omega), \\
\delta_{0}(\vec{q}, \Omega)=k_{0}^{\prime} \Omega+\frac{1}{2} k_{0}^{\prime \prime} \Omega^{2}+\rho_{0} q_{y}-\frac{1}{2 k_{0}}\left(q_{x}^{2}+q_{y}^{2}\right), \\
(j=1,2)
\end{gathered}
$$


the $z=0$ plane being taken at the input face of the crystal. In the following we shall assume that the pump pulse has a Gaussian profile both in space and time, of beam waist $w_{0}$ and time duration $\tau_{0}$ at $z=0$ :

$$
A_{0}(z=0, \vec{x}, t)=(2 \pi)^{3 / 2} A_{p} e^{-\left(x^{2}+y^{2}\right) / w_{0}^{2}} e^{-t^{2} / \tau_{0}^{2}}
$$

In Fourier space we have then the expression

$$
A_{0}(z=0, \vec{q}, \Omega)=2 \sqrt{2} \frac{A_{p}}{\delta q_{0}^{2} \delta \omega_{0}} e^{-\left(q_{x}^{2}+q_{y}^{2}\right) / \delta q_{0}^{2}} e^{-\Omega^{2} / \delta \omega_{0}^{2}},
$$

where

$$
\delta q_{0}=2 / w_{0}, \quad \delta \omega_{0}=2 / \tau_{0}
$$

denote the bandwidths of the pump in the spatial frequency domain and in the temporal frequency domain respectively.

Let us now consider the limit of the PWPA approximation, in which $w_{0}$ and $\tau_{0}$ tend to infinity and

$$
A_{0}(z, \vec{q}, \Omega) \rightarrow(2 \pi)^{3 / 2} A_{p} \delta(\vec{q}) \delta(\Omega)
$$

Under this condition, Eqs. (7) couple only pairs of phaseconjugated modes $(\vec{q}, \Omega)_{1}$ and $(-\vec{q},-\Omega)_{2}$ and can be solved analytically. The unitary input-output transformations relating the field operators at the output face of the crystal $a_{j}^{\text {out }}(\vec{q}, \Omega) \equiv a_{j}\left(z=l_{c}, \vec{q}, \Omega\right)$ to those at the input face $a_{j}^{i n}(\vec{q}, \Omega) \equiv a_{j}(z=0, \vec{q}, \Omega)$ take the following form:

$$
\begin{aligned}
& a_{1}^{\text {out }}(\vec{q}, \Omega)=U_{1}(\vec{q}, \Omega) a_{1}^{\text {in }}(\vec{q}, \Omega)+V_{1}(\vec{q}, \Omega) a_{2}^{i n \dagger}(-\vec{q},-\Omega), \\
& a_{2}^{\text {out }}(\vec{q}, \Omega)=U_{2}(\vec{q}, \Omega) a_{2}^{\text {in }}(\vec{q}, \Omega)+V_{2}(\vec{q}, \Omega) a_{1}^{i n \dagger}(-\vec{q},-\Omega),
\end{aligned}
$$

with

$$
\begin{aligned}
U_{1}(\vec{q}, \Omega)= & \exp \left[i \frac{\delta_{1}(\vec{q}, \Omega)-\delta_{2}(-\vec{q},-\Omega)-\Delta_{0}}{2} l_{c}\right] \\
& \times\left[\cosh \left[\Gamma(\vec{q}, \Omega) l_{c}\right]\right. \\
& \left.+i \frac{\Delta(\vec{q}, \Omega)}{2 \Gamma(\vec{q}, \Omega)} \sinh \left(\Gamma(\vec{q}, \Omega) l_{c}\right)\right], \\
V_{1}(\vec{q}, \Omega)= & \exp \left[i \frac{\delta_{1}(\vec{q}, \Omega)-\delta_{2}(-\vec{q},-\Omega)-\Delta_{0}}{2} l_{c}\right] \\
& \times \frac{\sigma_{p}}{\Gamma(\vec{q}, \Omega)} \sinh \left(\Gamma(\vec{q}, \Omega) l_{c}\right),
\end{aligned}
$$

$$
\begin{aligned}
U_{2}(\vec{q}, \Omega)= & \exp \left[i \frac{\delta_{2}(\vec{q}, \Omega)-\delta_{1}(-\vec{q},-\Omega)-\Delta_{0}}{2} l_{c}\right] \\
& \times\left[\cosh \left[\Gamma(-\vec{q},-\Omega) l_{c}\right]\right. \\
& \left.+i \frac{\Delta(-\vec{q},-\Omega)}{2 \Gamma(-\vec{q},-\Omega)} \sinh \left(\Gamma[-\vec{q},-\Omega] l_{c}\right)\right], \\
V_{2}(\vec{q}, \Omega)= & \exp \left[i \frac{\delta_{2}(\vec{q}, \Omega)-\delta_{1}(-\vec{q},-\Omega)-\Delta_{0}}{2} l_{c}\right] \\
& \times \frac{\sigma_{p}}{\Gamma(-\vec{q},-\Omega)} \sinh \left(\Gamma[-\vec{q},-\Omega] l_{c}\right),
\end{aligned}
$$

and

$$
\begin{gathered}
\Gamma(\vec{q}, \Omega)=\sqrt{\sigma_{p}^{2}-\frac{\Delta(\vec{q}, \Omega)^{2}}{4}} \\
\Delta(\vec{q}, \Omega)=\Delta_{0}+\delta_{1}(\vec{q}, \Omega)+\delta_{2}(-\vec{q},-\Omega) \approx k_{1 z}(\vec{q}, \Omega) \\
+k_{2 z}(-\vec{q},-\Omega)-k_{0}, \\
\sigma_{p}=\sigma A_{p} .
\end{gathered}
$$

It is important to note that the gain functions $U_{j}$ and $V_{j}$ given by Eq. (15) satisfy the following unitarity conditions:

$$
\begin{gathered}
\left|U_{j}(\vec{q}, \Omega)\right|^{2}-\left|V_{j}(\vec{q}, \Omega)\right|^{2}=1 \quad(j=1,2) \\
U_{1}(\vec{q}, \Omega) V_{2}(-\vec{q},-\Omega)=U_{2}(-\vec{q},-\Omega) V_{1}(\vec{q}, \Omega),
\end{gathered}
$$

which guarantee the conservation of the free-field commutation relations (4) after propagation.

\section{MEAN INTENSITY DISTRIBUTION}

In the following we shall consider measurements either in the near-field or in the far-field zones of the nonlinear crystal. In order to simplify the notation we shall omit the explicit dependence of the fields on the $z$ coordinate: when specification is explicitly needed, the measured quantities will be labeled with $\pi$ or $\pi^{\prime}$, which will denote the near-field and the far-field detection planes, respectively [see scheme of Fig. 1(a)]. The analytical results given here and in the next sections are all obtained within the PWPA: on the one hand, they generalize those illustrated in Ref. [17] for a type-I crystal at degeneracy to a type-II phase-matching configuration; on the other hand, they provide a good starting point to interpret the results of the numerical model that includes the pulse shape and the finite cross section of of the pump beam. A more general input-output formalism that goes beyond the PWPA is developed in Appendix A.

With a stationary and plane-wave pump the near-field intensity distribution in the output plane of the crystal clearly does not depend on $\vec{x}$ and $t$, because of the system invariance 

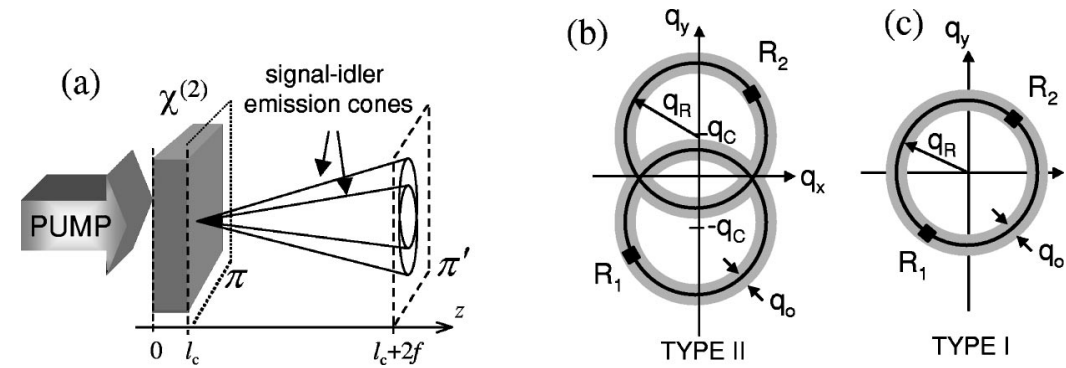

FIG. 1. Scheme for the observation of down-conversion in the far-field zone (a). The lens (not shown in the figure) is located at $z=l_{c}$ $+f$. (b) and (c) display the phase-matching curves (25) in the spatial frequency plane for a type-II (b) and a type-I (c) crystal, respectively. The symmetrical black squares $R_{1}$ and $R_{2}$ indicate the locations of the detectors from which maximal signal-idler correlation can be measured.

with respect to translation in time and in the transverse plane. Using input-output relations (14) and recalling that the input fields are the vacuum states, we obtain easily

$$
\left\langle I_{j}(\vec{x}, t)\right\rangle_{\pi}=\int \frac{d \Omega}{2 \pi} \int \frac{d \vec{q}}{(2 \pi)^{2}}\left|V_{j}(\vec{q}, \Omega)\right|^{2} \quad(j=1,2) .
$$

The function $\left|V_{j}(\vec{q}, \Omega)\right|^{2}$ gives the contribution of mode $(\vec{q}, \Omega)_{j}$ to the total photon flux of beam $j$, and is usually referred to as its spectral gain. On the other hand, in the far-field plane $\pi^{\prime}$ the spatial Fourier modes are resolved spatially and the photon distribution reflects the $\vec{q}$ dependence of these spectral functions. From the expression of $\Gamma(\vec{q}, \Omega)$ given by Eqs. (16), we see that down-conversion occurs most efficiently for the modes satisfying the condition $\Delta(\vec{q}, \Omega)$ $<2 \sigma_{p}$. Using Eqs. (16b) and (8), the phase mismatch accumulated during propagation can be written in the form

$\Delta(\vec{q}, \Omega) l_{c}=\Delta_{0} l_{c}+\operatorname{sgn}\left[k_{1}^{\prime}-k_{2}^{\prime}\right] \frac{\Omega}{\Omega_{0}^{\prime}}+\frac{\Omega^{2}}{\Omega_{0}^{\prime 2}}-\rho_{2} q_{y}-\frac{q_{x}^{2}+q_{y}^{2}}{q_{0}^{2}}$,

where we assumed that the signal wave is ordinarily polarized, so that $\rho_{1}=0$, and we introduced the parameters

$$
q_{0}=\sqrt{\frac{\bar{k}}{l_{c}}}, \quad \Omega_{0}^{\prime}=\frac{1}{\left|k_{1}^{\prime}-k_{2}^{\prime}\right| l_{c}}, \quad \Omega_{0}^{\prime \prime}=\sqrt{\frac{2}{\left(k_{1}^{\prime \prime}+k_{2}^{\prime \prime}\right) l_{c}}},
$$

where $\bar{k}=2 k_{1} k_{2} /\left(k_{1}+k_{2}\right)$. These determine the characteristic bandwidths of PDC both in the temporal frequency domain and in spatial frequency domain. In the type-I phasematching configuration we will consider in Sec. VI A, both the signal and the idler waves are ordinarily polarized and are observed close to degeneracy, i.e., for $\omega_{1}=\omega_{2}=\omega_{0} / 2$. In this special case the temporal bandwidth is determined by $\Omega_{0} \equiv \Omega_{0}^{\prime \prime}=\sqrt{1 /\left|k_{1}^{\prime \prime} l_{c}\right|}$, since $k_{1}(\omega)=k_{2}(\omega)$ implies that $\Omega_{0}^{\prime}$ $=\infty$. Far from frequency degeneracy the emission spectrum has a much narrower bandwidth, on the order of $\Omega_{0} \equiv \Omega_{0}^{\prime}$ which is about two to three orders of magnitude smaller than $\Omega_{0}^{\prime \prime}$ (for a typical crystal length of few millimeters). On the other hand, in type-II crystals the signal and idler waves are characterized by different polarizations and frequency dispersion relations, so that $\Omega_{0} \equiv \Omega_{0}^{\prime}$ remains finite even for $\omega_{1}=\omega_{2}$. The spatial bandwidth $q_{0}$ gives the range of transverse wave vectors for which the gain spectrum $\left|V_{j}(\vec{q}, \Omega)\right|^{2}$ is close to its maximum value, $\sinh ^{2}\left(\sigma_{p} l_{c}\right)$, when the $j$ th field is observed at a given frequency $\omega_{j}+\Omega$ [the gray region shown schematically in Figs. 1(b) and 1(c) for $\Omega=0$ ]. We remark that $1 / \rho_{2} l_{c}$ and $q_{0}$ are about the same order of magnitude as long as $l_{c}$ remains in the millimeter range.

For definiteness we assume that the far field is observed in the focal plane of a thin lens of focal length $f$ which performs the Fourier transformation of the field from the output face of the crystal (the so-called $f-f$ system). The field operators in the focal plane $\pi^{\prime}$ at $z=l_{c}+2 f$ [see Fig. 1(a)], which we denote with $b_{1,2}(\vec{x}, t)$, are related to those in the output plane of the crystal by the following Fresnel transformation:

$$
\begin{gathered}
b_{j}(\vec{x}, t)=\int d \vec{x}^{\prime} h_{j}\left(\vec{x}, \vec{x}^{\prime}\right) a_{j}^{\text {out }}\left(\vec{x}^{\prime}, t\right), \\
h_{j}\left(\vec{x}, \vec{x}^{\prime}\right)=\frac{-i}{\lambda_{j} f} \exp ^{-\left(2 \pi i / \lambda_{j} f\right) \vec{x} \cdot \vec{x}^{\prime}} \quad(j=1,2),
\end{gathered}
$$

where $\lambda_{j}=2 \pi c / \omega_{j}(j=1,2)$ are the free-space wavelengths corresponding to the carrier frequencies. Using the inputoutput relations (14) and unitarity relations (17) we can evaluate the mean intensity distribution of the two fields with the following approximate expression:

$$
\left\langle I_{j}(\vec{x}, t)\right\rangle_{\pi^{\prime}} \approx \frac{1}{S_{\text {diff }}^{(j)}} \int \frac{d \Omega}{2 \pi}\left|\bar{V}_{j}(\vec{x}, \Omega)\right|^{2} \quad(j=1,2),
$$

where we introduced the barred gain functions defined in real space

$$
\begin{gathered}
\bar{U}_{j}(\vec{x}, \Omega)=U_{j}\left(\frac{2 \pi}{\lambda_{j} f} \vec{x}, \Omega\right), \quad \bar{V}_{j}(\vec{x}, \Omega)=V_{j}\left(\frac{2 \pi}{\lambda_{j} f} \vec{x}, \Omega\right), \\
(j=1,2),
\end{gathered}
$$

and $S_{\text {diff }}^{(j)}=\left(\lambda_{j} f\right)^{2} / S_{A}(j=1,2)$, denotes the resolution areas in the far-field plane at the S-I wavelengths, with $S_{A}$ being the area characterizing the dimension of the system in the 
transverse plane. As is shown in Ref. [17], Eq. (22) can be obtained by assuming that a pupil of area $S_{A} \gg 1 / q_{0}^{2}$ is put on the crystal exit face. Assuming that the transverse dimensions of the crystal are large compared to the pump waist, $S_{A}$ can be identified with the effective cross section area of the pump beam. Equation (22) represents a good approximation provided the pump beam shape changes negligibly during propagation in the crystal and behaves therefore as a plane wave. This happens when the Rayleigh length characterizing the Gaussian pump beam divergence, $z_{R}^{0}=\pi w_{0}^{2} / \lambda_{0}$, and its analog characterizing dispersion, $z_{\text {disp }}^{0}=\tau_{0}^{2} / 2 k_{0}^{\prime \prime}$, are much longer than the crystal length $l_{c}$. The same conditions can also be written in terms of the pump spatial and temporal bandwidths (12) as

$$
\frac{\delta q_{0}}{q_{0}} \ll 1, \quad \frac{\delta \omega_{0}}{\Omega_{0}} \ll 1
$$

At the considered carrier frequencies $\omega_{1}$ and $\omega_{2}$ the gain functions $V_{j}(\vec{q}, \Omega=0)$ are maximal and perfect phase matching is achieved when the equations $\Delta( \pm \vec{q}, \Omega=0)=0$ are satisfied, with the plus sign for field 1 , and the minus sign for field 2 [see Eqs. (15) and (16b)]. More explicitly, as can be seen using expression (19), they can be written as

$$
\frac{q_{x}^{2}}{q_{0}^{2}}+\left(\frac{q_{y}}{q_{0}} \pm \frac{1}{2} \rho_{2} l_{c} q_{0}\right)^{2}=\Delta_{0} l_{c}+\left(\frac{1}{2} \rho_{2} l_{c} q_{0}\right)^{2} .
$$

Provided that $\Delta_{0} l_{c}>-\frac{1}{4} \rho_{2}^{2} l_{c}^{2} q_{0}^{2}$, we have therefore two circles of radius $q_{R}$ and centered at $\left(q_{x}=0, q_{y}= \pm q_{C}\right)$, with

$$
\begin{gathered}
q_{C}=\frac{1}{2} \rho_{2} l_{c} q_{0}^{2}=\frac{1}{2} \bar{k} \rho_{2}, \\
q_{R}=q_{0} \sqrt{\Delta_{0} l_{c}+\frac{q_{C}^{2}}{q_{0}^{2}}}=\sqrt{\bar{k} \Delta_{0}+\frac{1}{4}\left(\bar{k} \rho_{2}\right)^{2}} .
\end{gathered}
$$

They are plotted in Figs. 1(b,c), respectively, for a type-II and a type-I phase-matching configuration. Modes close to these circles within $q_{0}$ (gray annuli in the figure) give a non-negligible contribution to the down-converted field. In the detection plane they give rise to characteristic couples of rings which have been observed in many experiments on parametric down-conversion (see, e.g., Refs. [25-27]). It should be stressed that, without any spectral filtering, emission occurs on a very wide range of wavelengths and emission angles, as allowed by the phase-matching conditions (see, e.g., Refs. [26,9]). However, from an experimental point of view, a particular couple of rings can always be selected with the use of frequency filters centered at the chosen frequencies $\omega_{1}$ and $\omega_{2}=\omega_{0}-\omega_{1}$. Noting that there is the following mapping between the spatial frequency plane and the far field plane:

$$
\left(q_{x}, q_{y}\right) \rightarrow \frac{\lambda_{1} f}{2 \pi}\left(q_{x}, q_{y}\right) \text { for field } 1
$$

(a)

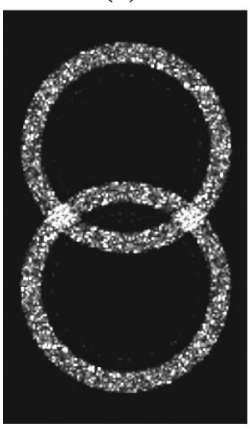

$\Delta_{0}>0$ (b)

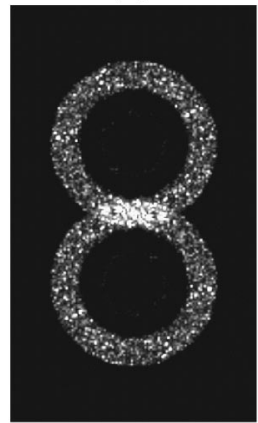

$\Delta_{0}=0$ (c)

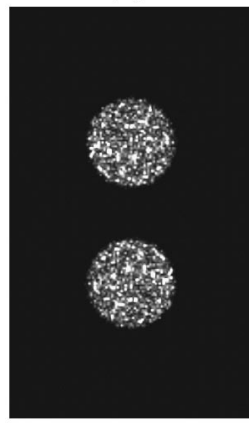

$\Delta_{0} l_{c}=-q_{C}^{2} / q_{0}^{2}$
FIG. 2. Typical far-field pattern from down-conversion in a type-II crystal, assuming observation is performed at the degenerate frequency (i.e., at $\lambda_{1}=\lambda_{2}$ ). They are obtained for decreasing values of the collinear phase-mismatch parameter $\Delta_{0}$, which makes the radius of the rings shrinks to zero. The pump pulse duration is $\tau_{0}$ $=1.5 \mathrm{ps}$, the pump beam waist is $w_{0}=664 \mu \mathrm{m}\left(\delta q_{0} / q_{0}=0.05\right)$, and the parametric gain is $\sigma_{p} l_{c}=4$.

$$
\left(q_{x}, q_{y}\right) \rightarrow \frac{\lambda_{2} f}{2 \pi}\left(q_{x}, q_{y}\right) \quad \text { for field } 2,
$$

it is easily seen that the ring radii, $x_{R}^{(1,2)}=\lambda_{1,2} f / 2 \pi q_{R}$, and their distance from the $z$ axis, $y_{C}^{(1,2)}=\lambda_{1,2} f / 2 \pi q_{C}$, are generally different except when observation is performed at frequency degeneracy (i.e., for $\lambda_{1}=\lambda_{2}$ ). In a type-I phasematching configuration these rings are concentric, since there is no spatial walk-off between the two fields $\left(\rho_{2}=0\right)$ and the radial symmetry of the system is preserved.

Figures 2 and 3 illustrate the kind of far-field patterns that can be obtained from a single pump pulse at frequency degeneracy in a type-I and a type-II crystal, respectively. They are obtained by numerical integration of the classical field equations (2), with a white input noise which simulates the vacuum fluctuations that trigger the process, as will be described in Sec. VI. The pump pulse duration is $1.5 \mathrm{ps}$ and the large waist condition $\delta q_{0} \ll q_{0}$ is fulfilled. In the type-II case the width of the rings is determined by the interval of frequencies of the numerical grid. In the examples shown in Fig. 2 the grid acts as a $15 \mathrm{~nm}$ box-shaped interference filter. In the type-I phase-matching case, being at frequency degen- (a)

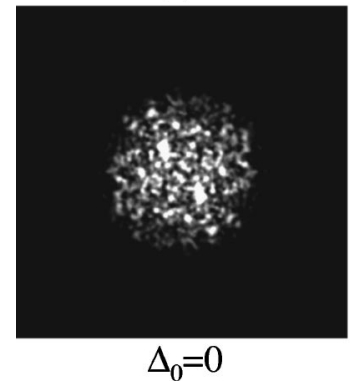

(b)

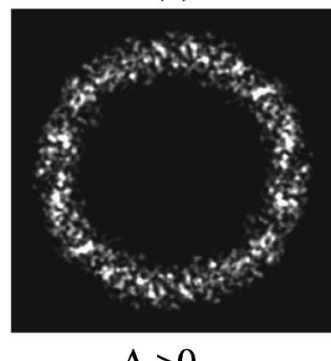

$\Delta_{0}>0$
FIG. 3. Far-field pattern in a type-I crystal at the degenerate frequency for collinear (a) and noncollinear (b) phase-matching. $\tau_{0}=1.5 \mathrm{ps}, \delta q_{0} / q_{0}=0.05\left(w_{0}=920 \mu \mathrm{m}\right)$ and $\sigma_{p} l_{c}=4$. 


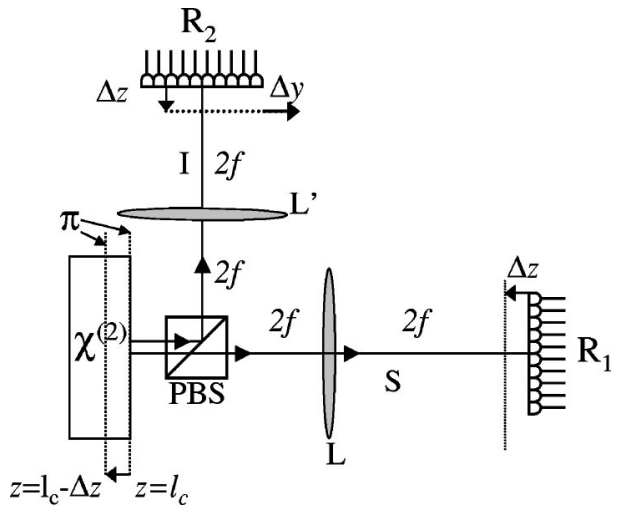

FIG. 4. Detection scheme to measure spatial correlations in the near field. A polarizing beam splitter (PBS) separates the S-I beams. Their near fields, at the plane $\pi: z=l_{c}-\Delta z$, are imaged by two lenses ( $L$ and $L^{\prime}$ ) onto the pixel detectors $R_{1}$ and $R_{2}$, which lie in the plane conjugate to plane $\pi . \Delta z$ and $\Delta y$ indicate the spatial shifts applied to the optical devices that are necessary to optimize the measurement.

eracy, the two rings merge into one that contains both signal and idler modes. Moreover, in this case the width of the rings in the spatial frequency plane is determined by the natural bandwidth $q_{0}$, since the temporal frequency interval used in the numerical simulations is small compared to the characteristic temporal bandwidth $\Omega_{0}$ [as pointed out in the discussion following Eqs. (20), in the type-I configuration at degeneracy the temporal bandwidth is much larger than in the type-II configuration].

\section{NEAR-AND FAR-FIELD CORRELATIONS}

We now define explicitly the quantities that can be measured in an experiment in order to put in evidence the S-I correlations in the spatial domain we are investigating. We assume that the signal and idler beams are spatially separated in the detection plane and are measured over two detection areas, which we denote with $R_{1}$ and $R_{2}$.

In the far field, correlations find their origin in the conservation of the transverse momentum of the generated photon pairs. Therefore, in order to find maximal correlation, $R_{1}$ and $R_{2}$ must correspond to couples of phase-conjugate modes, such as those indicated with the black squares in Figs. 1(b) and $1(\mathrm{c})$. For simplicity, in order to avoid the heavy notations that arise if $\lambda_{1} \neq \lambda_{2}$, we shall restrict our analysis to the frequency degenerate case, indicating with $\lambda$ both $\lambda_{1}$ and $\lambda_{2}$. Phase-conjugate modes are then mapped by the lens into symmetrical points in plane $\pi^{\prime}$ according to relation (27) and $R_{1}$ and $R_{2}$ must be taken symmetrical.

On the other side, near-field correlations arising from the position entanglement of the twin photons are expected to be observed if $R_{1}$ and $R_{2}$ occupy the same region of the nearfield plane. In practice a type-II phase-matching configuration should be considered, so that the use of a polarizing beam splitter and lens systems allows the imaging of the S-I near fields on two physically separated detection planes (see detection scheme illustrated in Fig. 4).

If the detectors are the pixels of a CCD camera, as in the experiment described in Ref. [26], they do not allow any spectral measurement due to the very low resolution power of the device in the time domain. They simply measure the total number of incoming photons down-converted in each single pump shot, and the measurement time $T_{d}$ can be identified with the pump pulse duration. We introduce therefore the operators corresponding to the number of photons collected by the two detectors in the finite-time window $\left[-T_{d} / 2, T_{d} / 2\right]$ :

$$
N_{j}=\int_{R_{j}} d \vec{x} \int_{-T_{d} / 2}^{T_{d} / 2} d t \quad I_{j}(\vec{x}, t) \quad(j=1,2) .
$$

The measurable quantity capable of displaying the quantum nature of the photon-number statistics in the spatial domain is the variance of the photon-number difference, $N_{-}=N_{1}$ $-N_{2}$, which can be written in the form

$$
\left\langle\left(\delta N_{-}\right)^{2}\right\rangle=\left\langle N_{+}\right\rangle+\left\langle:\left(\delta N_{1}\right)^{2}:\right\rangle+\left\langle:\left(\delta N_{2}\right)^{2}:\right\rangle-2\left\langle\delta N_{1} \delta N_{2}\right\rangle .
$$

$\delta N_{j}=N_{j}-\left\langle N_{j}\right\rangle$ and $\delta N_{-}=N_{-}-\left\langle N_{-}\right\rangle$denote the photonnumber fluctuation operators associated with $N_{j}$ and $N_{-}$and the colon ":" denotes normal ordering (n.o.) for the expectation values. In Eq. (29) the shot-noise contribution, i.e., the total number of photons intercepted by the two detector $\left\langle N_{+}\right\rangle=\left\langle N_{1}\right\rangle+\left\langle N_{2}\right\rangle$, has been explicitly separated from the term that describes the field correlations. We define

$$
\begin{aligned}
\left\langle: \delta N_{i} \delta N_{j}:\right\rangle= & \int_{R_{i}} d \vec{x} \int_{R_{j}} d \vec{x} \int_{-T_{d} / 2}^{T_{d} / 2} d t \int_{-T_{d} / 2}^{T_{d} / 2} \\
& \times d t^{\prime} G_{i j}\left(\vec{x}, t, \vec{x}^{\prime}, t^{\prime}\right) \quad(i, j=1,2),
\end{aligned}
$$

where

$$
\begin{aligned}
G_{i j}\left(\vec{x}, t, \vec{x}^{\prime}, t^{\prime}\right)= & \left\langle: I_{i}(\vec{x}, t) I_{j}\left(\vec{x}^{\prime}, t^{\prime}\right):\right\rangle-\left\langle I_{i}(\vec{x}, t)\right\rangle \\
& \times\left\langle I_{j}\left(\vec{x}^{\prime}, t^{\prime}\right)\right\rangle \quad(i, j=1,2)
\end{aligned}
$$

are the n.o. self- and cross-photon number correlation functions of the S-I beams. Notice that in the nondegenerate case (type II or type I far from frequency degeneracy) we have $\left\langle: I_{1}(\vec{x}, t) I_{2}\left(\vec{x}^{\prime}, t^{\prime}\right):\right\rangle=\left\langle I_{1}(\vec{x}, t) I_{2}\left(\vec{x}^{\prime}, t^{\prime}\right)\right\rangle$.

We now focus on the analytical results that can be deduced from the PWPA. We shall consider explicitly only the case of type-II phase matching. The case of type I, at least in the far field, can be described with a similar treatment and has been already discussed in Ref. [17]. Assuming that the detection time $T_{d}$ is large compared to the coherence time $\tau_{c o h}=\Omega_{0}^{-1}$, as is usually the case, we have

$$
\left\langle: \delta N_{i} \delta N_{j}:\right\rangle \approx T_{d} \int_{R_{i}} d \vec{x} \int_{R_{j}} d \vec{x}^{\prime} G_{i j}\left(\vec{x}, \vec{x}^{\prime}, \Omega=0\right) \quad(i, j=1,2),
$$

where $G_{i j}\left(\vec{x}, \vec{x}^{\prime}, \Omega=0\right)$ is the Fourier transform of function (31) with respect to $t-t^{\prime}$ (notice that in a cw regime this 
function depends only on $\left.t-t^{\prime}\right)$. Next, the Gaussian character of the field statistics allows to express fourth-order correlations in terms of second-order correlations [see Appendix A, Eq. (A5b)] In this way the photon-number correlation defined by Eq. (30) in a plane $z$ can be written as the photonnumber correlations defined by Eq. (30) in a plane $z$ as

$$
\begin{aligned}
\left\langle: \delta N_{i} \delta N_{j}:\right\rangle_{z}= & T_{d} \int_{R_{i}} d \vec{x} \int_{R_{j}} d \vec{x}^{\prime} \int \frac{d \Omega}{2 \pi}\left|\Gamma_{i j}^{(z)}\left(\vec{x}, \vec{x}^{\prime}, \Omega\right)\right|^{2} \\
& (i, j=1,2)
\end{aligned}
$$

where

$$
\begin{gathered}
\Gamma_{j j}^{(z)}\left(\vec{x}, \vec{x}^{\prime}, \Omega\right)=\int d \tau e^{-i \Omega \tau}\left\langle a_{j}^{\dagger}(z, \vec{x}, t+\tau) a_{j}\left(z, \vec{x}^{\prime}, t\right)\right\rangle \\
(j=1,2), \\
\Gamma_{12}^{(z)}\left(\vec{x}, \vec{x}^{\prime}, \Omega\right)=\int d \tau e^{-i \Omega \tau}\left\langle a_{1}(z, \vec{x}, t+\tau) a_{2}\left(z, \vec{x}^{\prime}, t\right)\right\rangle,
\end{gathered}
$$

are the only correlation spectra of the S-I fields which do not vanish when the input field is in the vacuum state. We assumed here implicitly that propagation in free space occurs without losses.

In the far-field plane $\pi^{\prime}$, the self- and cross-correlation functions are given by

$$
\begin{gathered}
\Gamma_{j j}^{\left(\pi^{\prime}\right)}\left(\vec{x}, \vec{x}^{\prime}, \Omega\right)=\delta\left(\vec{x}-\vec{x}^{\prime}\right)\left|\bar{V}_{j}(\vec{x}, \Omega)\right|^{2} \quad(j=1,2) \\
\Gamma_{12}^{\left(\pi^{\prime}\right)}\left(\vec{x}, \vec{x}^{\prime}, \Omega\right)=-\delta\left(\vec{x}+\vec{x}^{\prime}\right) \bar{U}_{1}(\vec{x}, \Omega) \bar{V}_{2}(-\vec{x},-\Omega)
\end{gathered}
$$

as can be inferred using the Fresnel transformations (21). In this case, both correlation functions display a $\delta$-like peak, located at $\vec{x}^{\prime}=\vec{x}$ for the self-correlation, and at $\vec{x}^{\prime}=-\vec{x}$ for the cross correlation. The $\delta$-like character of the correlations derives from the unphysical assumption that the transverse dimensions of the system are infinite. In Ref. [17], which deals only with far-field correlations, the transverse size of the system was taken into account $a$ posteriori by considering a finite aperture $S_{A}$ placed at the crystal output face, assuming a condition equivalent to Eq. (24) is met. With this approach, we found that far-field correlations are localized within the resolution area determined by the size of this aperture [that is, $S_{\text {diff }}=(\lambda f)^{2} / S_{A}$ with the $f$ - $f$ lens system]. This procedure also eliminates the cumbersome divergencies arising from the singularity of the spatial $\delta$ functions, allowing the formal substitution $\delta(\vec{x}=0) \rightarrow 1 / S_{\text {diff }}$ as has been done in Eq. (22). By integrating over two symmetric detection pixels of area much larger than this resolution length, we obtain

$$
\begin{aligned}
\left\langle:\left(\delta N_{1}\right)^{2}:\right\rangle_{\pi^{\prime}} & =\left\langle:\left(\delta N_{2}\right)^{2}:\right\rangle_{\pi^{\prime}} \\
& =\frac{1}{S_{d i f f}} \int \frac{d \Omega}{2 \pi} \int_{R_{1}} d \vec{x}\left|\bar{V}_{1}(\vec{x}, \Omega)\right|^{4},
\end{aligned}
$$

$$
\left\langle\delta N_{1} \delta N_{2}\right\rangle_{\pi^{\prime}}=\frac{1}{S_{d i f f}} \int \frac{d \Omega}{2 \pi} \int_{R_{1}} d \vec{x}\left|\bar{U}_{1}(\vec{x}, \Omega) \bar{V}_{2}(-\vec{x},-\Omega)\right|^{2} .
$$

In order to obtain these expressions, both unitarity relations (17a) and (17b) must be used, together with the fact that the integration areas $R_{1}$ and $R_{2}$ are taken symmetric with respect to the origin. We then easily get

$$
\begin{aligned}
& \left\langle:\left(\delta N_{1}\right)^{2}:\right\rangle_{\pi^{\prime}}+\left\langle:\left(\delta N_{2}\right)^{2}:\right\rangle_{\pi^{\prime}}-2\left\langle\delta N_{1} \delta N_{2}\right\rangle_{\pi^{\prime}} \\
& \quad=-\frac{1}{S_{d i f f}} \int \frac{d \Omega}{2 \pi} \int_{R_{1}} d \vec{x}\left|\bar{V}_{1}(\vec{x}, \Omega)\right|^{2}, \\
& \quad=-\left\langle N_{+}\right\rangle_{\pi^{\prime}},
\end{aligned}
$$

which implies $\left\langle\left(\delta N_{-}\right)^{2}\right\rangle_{\pi^{\prime}}=0$, as follows from Eq. (29). A more rigorous approach to the issue concerning the finite resolution of the system is given in Appendix B, where we derive an approximate solution of the propagation equations which include the finite pump dimensions in limit (24). It is shown that the widths of the far-field correlation peaks are indeed on the order of $x_{\text {diff }}=(\lambda f / 2 \pi) \delta q_{0}$, the resolution length determined by the pump beam waist $w_{0}$.

In the near-field plane $\pi$ (at $z=l_{c}$ ), using Eqs. (14) and (4) we obtain the expressions

$$
\Gamma_{j j}^{(\pi)}\left(\vec{x}, \vec{x}^{\prime}, \Omega\right)=\int \frac{d \vec{q}}{(2 \pi)^{2}} e^{-i \vec{q} \cdot\left(\vec{x}-\vec{x}^{\prime}\right)}\left|V_{j}(\vec{q}, \Omega)\right|^{2} \quad(j=1,2),
$$

$$
\begin{aligned}
\Gamma_{12}^{(\pi)}\left(\vec{x}, \vec{x}^{\prime}, \Omega\right)= & \int \frac{d \vec{q}}{(2 \pi)^{2}} e^{i \vec{q} \cdot\left(\vec{x}-\vec{x}^{\prime}\right)} \\
& \times U_{1}(\vec{q}, \Omega) V_{2}(-\vec{q},-\Omega),
\end{aligned}
$$

which depend only on the offset between the two points, $\vec{x}$ $-\vec{x}^{\prime}$, as a consequence of the invariance of the system with respect to translations in the transverse plane which follows from the PWPA. Provided that the typical scale of variation of the function appearing under the integrals in Eqs. (38a) and (38b) is $q_{0}$, we expect that these correlations are localized in a region of size

$$
x_{c o h} \equiv 1 / q_{0} \approx \sqrt{l_{c} / \bar{k}},
$$

a quantity that can be identified with the transverse coherence length of the down-converted fields. This finite correlation length comes from the spread out of the generated photons due to diffraction, which increases proportionally to the square root of the propagation distance; we can therefore expect that the detection areas must be larger than this coherence area in order to measure good correlations in the near field.

We incidentally note that the cross-correlation function $\Gamma_{12}$ displays a localized peak, which in the near field is located at $\vec{x}^{\prime}=\vec{x}$ [Eq. (38b)], while in the far field it is located at $\vec{x}^{\prime}=-\vec{x}$ [Eq. (35b)]. The $\vec{x} \leftrightarrow \vec{x}^{\prime}$ correlation in the near 
field reflects the entanglement in positions of the twin photons, while the $\vec{x} \leftrightarrow-\vec{x}^{\prime}$ correlation in the far field comes from their entanglement in momentum.

For what concerns the near field, let us consider more in general what happens in a generic plane of coordinate $z$ close to the crystal output face. As is shown schematically in Fig. 4 , we consider a measurement in which the signal and the idler fields are separated with a polarizing beam splitter placed beyond the crystal. The two lenses $L$ and $L^{\prime}$ put in the signal and idler arms perform the imaging of plane $z$ onto two distinct detection planes (note that we have here two $2 f-2 f$ lens systems, while in the case of the far-field measurement considered previously we had a single $f$ - $f$ system). The S-I photons are collected by means of two square pixel detectors $R_{1}$ and $R_{2}$, centered at positions $\vec{x}_{1}$ and $\vec{x}_{2}$, respectively. The propagation from the crystal exit face $z=l_{c}$ to the detection planes can be described by a Fresnel transformation of form (21a) with the kernel

$$
h\left(\vec{x}, \vec{x}^{\prime}\right)=\frac{-i}{\lambda\left(z-l_{c}\right)} \exp \left(\frac{-i}{\lambda\left(z-l_{c}\right)}\left|\vec{x}-\vec{x}^{\prime}\right|^{2}\right),
$$

where inessential phase factors due to the presence of the lenses have been omitted. By using this transformation inside Eqs. (34a) and (34b) we can calculate explicitly each term on the rhs of Eq. (29). By performing explicitly the integration over the square pixel areas in Eq. (33), we obtain

$$
\begin{gathered}
\left\langle:\left(\delta N_{j}\right)^{2}:\right\rangle_{z}=T_{d} \int d \vec{q} \int d \vec{q}^{\prime} H_{11}\left(\vec{q}, \vec{q}^{\prime}\right) \\
\quad \times \int \frac{d \Omega}{2 \pi}\left|V_{j}(\vec{q}, \Omega)\right|^{2}\left|V_{j}\left(\vec{q}^{\prime}, \Omega\right)\right|^{2} \\
(j=1,2), \\
\left\langle\delta N_{1} \delta N_{2}\right\rangle_{z}=T_{d} \int d \vec{q} \int d \vec{q}^{\prime} H_{12}\left(\vec{q}, \vec{q}^{\prime}\right) \int \frac{d \Omega}{2 \pi} \bar{U}_{1}(\vec{q}, \Omega) \\
\times \bar{V}_{2}(-\vec{q},-\Omega) \bar{U}_{1}^{*}\left(\vec{q}^{\prime}, \Omega\right) \bar{V}_{2}^{*}\left(-\vec{q}^{\prime},-\Omega\right), \\
\left\langle N_{+}\right\rangle_{z}=2 T_{d} d^{2} \int \frac{d \vec{q}}{(2 \pi)^{2}} \int \frac{d \Omega}{2 \pi}\left|V_{j}(\vec{q}, \Omega)\right|^{2},
\end{gathered}
$$

where the functions $H_{i j}$ depend on the square pixel size $d$ and on their relative positions through the relations

$$
\begin{gathered}
H_{11}\left(\vec{q}, \vec{q}^{\prime}\right)=(d / 2 \pi)^{4} \operatorname{sinc}^{2}\left[\frac{\left(q_{x}-q_{x}^{\prime}\right) d}{2}\right] \operatorname{sinc}^{2}\left[\frac{\left(q_{y}-q_{y}^{\prime}\right) d}{2}\right], \\
H_{12}\left(\vec{q}, \vec{q}^{\prime}\right)=\exp \left(-i \frac{\lambda\left(z-l_{c}\right)}{2 \pi}\left(q^{2}-q^{\prime 2}\right)\right. \\
\left.+i\left(\vec{q}-\vec{q}^{\prime}\right) \cdot\left(\vec{x}_{1}-\vec{x}_{2}\right)\right) H_{11}\left(\vec{q}, \vec{q}^{\prime}\right) . \quad(42 \mathrm{~b})
\end{gathered}
$$

We first note that if the detection areas are reduced well below the coherence area $x_{c o h}^{2}$, the fluctuations of $N_{-}$approach shot noise. Indeed, in the limit $d \ll x_{c o h}$ we can replace $H_{11}\left(\vec{q}, \vec{q}^{\prime}\right)$ with $H_{11}(\vec{q}, \vec{q})=d^{4} /(2 \pi)^{4}$ in Eqs. (41a) and (41b), from which it can be verified that the correlation terms scales as $d^{4} / x_{c o h}^{4}$ while the shot-noise contribution scale as $d^{2} / x_{c o h}^{2}$ (to evaluate this scaling it should be noted that for a fixed $\Omega$, the area in $\vec{q}$ space where the gain functions are not negligible is on the order of $\left.q_{0}^{2}=1 / x_{c o h}^{2}\right)$. On the other hand, if the detection areas are large enough with respect to $x_{c o h}^{2}$, the substitutions

$$
H_{i j}\left(\vec{q}, \vec{q}^{\prime}\right) \rightarrow \frac{d^{2}}{(2 \pi)^{2}} \delta\left(\vec{q}-\vec{q}^{\prime}\right)
$$

can be used for evaluating both Eq. (41a) and Eq. (41b); this leads to vanishing fluctuations in the measurement of $N_{-}$as for the far-field case [again, unitarity relations (17) must be used in order to obtain this result]. However, the condition large enough is more stringent for cross correlation than for self-correlation. Let us first consider the case $z=l_{c}$ and $\vec{x}_{1}$ $=\vec{x}_{2}$. The function appearing under the integral in Eq. (41a) is always positive. By contrast, the function appearing under the integral in Eq. (41b) is an oscillating function, which becomes positive only for an infinite pixel size, when limit (43) is strictly achieved. This feature tends to lower $\left\langle\delta N_{1} \delta N_{2}\right\rangle_{z}$ with respect to $\left\langle:\left(\delta N_{1}\right)^{2}:\right\rangle_{z}$ and $\left\langle:\left(\delta N_{2}\right)^{2}:\right\rangle_{z}$. As a consequence of this behavior the fluctuations of $N_{-}$will therefore exceed shot noise, as it can be easily inferred from expression (29). As a matter of fact, as we shall see in Sec. VI C, $\vec{x}_{1}=\vec{x}_{2}$ and $z=l_{c}$ are not the better choices to minimize the fluctuations of $N_{-}$, and special care in the positioning of the detectors is necessary in order to compensate both the effect of diffraction and the spatial walk-off between the S-I fields, which are included by the phase of the function $U_{1}(\vec{q}, \Omega) V_{2}(-\vec{q},-\Omega)$.

Finally we note that if the losses of the detection process are taken into account, the ideal result $\left\langle\left(\delta N_{-}\right)^{2}\right\rangle=0$ must be replaced with

$$
\left\langle\left(\delta N_{-}\right)^{2}\right\rangle=\eta(1-\eta)\left\langle N_{+}\right\rangle,
$$

$\eta$ denoting the finite quantum efficiency of the detectors.

\section{NUMERICAL RESULTS}

We now present the results obtained from the numerical model that includes the effects of the finite pump. The quantum averages in which we are interested (i.e., mean photon numbers and photon-number correlations) are evaluated through a stochastic method based on the Wigner representation. With respect to other representations in phase space, the Wigner representation presents the advantage that the $c$-number stochastic equations equivalent to the equations for the field operators (2) do not contain Langevin noise terms (because of linearity and the absence of dissipation) and are therefore identical to the classical propagation equations (7). The statistical character of the quantum fields is therefore 
wholly contained in the stochastic input field (see, e.g., Ref. [28] for more detailed discussion). We therefore proceed as follows:

(1) We generate the input field with the appropriate phasespace probability distribution, which is a Gaussian white noise with zero mean, corresponding to the vacuum state in the Wigner representation [29].

(2) We perform the numerical integration of Eqs. (7). We use a split-step algorithm [30] which integrates separately the terms describing linear propagation and the term describing the wave-mixing process: the former are integrated in Fourier space, the latter in real space.

(3) The obtained output fields are used to evaluate the correlation functions of interest. The procedure must be reiterated a sufficiently large number of times, so that the stochastic averages performed become good approximations to the corresponding quantum expectation values. Furthermore, some corrections are usually necessary in order to convert them to the desired operator ordering (the Wigner representation yields quantum expectation values of symmetrized operator products).

\section{A. Far-field correlation in a type-I crystal at degeneracy}

We first focus our attention on far-field correlations that can be observed in a type-I crystal with emission close to degeneracy (the case illustrated in Fig. 3). Since there is no spatial and temporal walk-off between the signal and the idler modes, the most significant parameters in play are the spatial bandwidth $q_{0}=\sqrt{\bar{k} / l_{c}}$ and the the ring radius $q_{R}$ $=\sqrt{\bar{k} \Delta_{0}}$, which are determined by the crystal length and the collinear phase-mismatch parameter [see Eqs. (20) and (26b)]. To a large extent, the analysis that we have performed does not depend on the particular type-I crystal considered. We shall, however, refer to the specific case of LBO $\left(\mathrm{LiB}_{3} \mathrm{O}_{5}\right.$ : lithium triborate) set in the type-I configuration described, e.g., in Refs. [25,26]. The pump field operates at $\lambda_{0}=532 \mathrm{~nm}$ and propagates in the $X Y$ crystal plane forming an angle with the $X$ axis close to $11^{\circ}$, for which collinear phase matching at degeneracy is achieved. In order to evaluate the characteristic bandwidths and walk-off parameters given by Eq. (20), we have used the Sellmeier dispersion relation coefficients, which can be found in Ref. [23]. Considering a crystal length of $5 \mathrm{~mm}$, the coherence time is $\tau_{c o h}=\Omega_{0}^{-1} \approx 0.01 \mathrm{ps}$. Assuming the pump pulse has a duration of about $1.5 \mathrm{ps}$, as in the experiment described in Ref. [26], the ratio $\delta \omega_{0} / \Omega_{0}$ is as small as $10^{-2}$. The analytical results obtained within the plane-wave and cw pump approximation are therefore expected to provide good insight as long as the ratio $\delta q_{0} / q_{0}$ remains small compared to unity. The temporal/spatial walk-off between the signal and the pump field is negligible unless the pump pulse time/beam waist is exceedingly small, a situation we do not consider, since it is too far from the ideal plane-wave pump limit (it would prevent the observation of any spatial quantum correlation effects).

Figure 5 displays the intensity distribution obtained numerically from a single integration of the propagation equations, for increasing values of the pump beam spatial band-
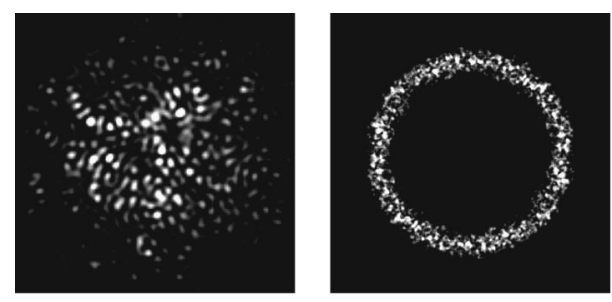

(a)
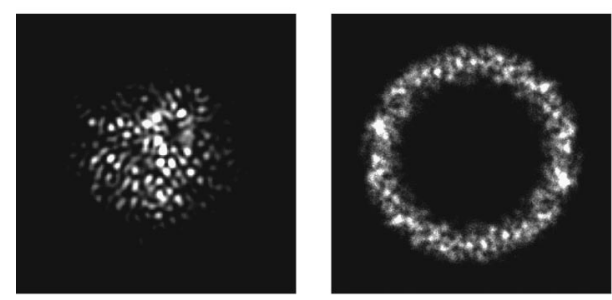

(b)
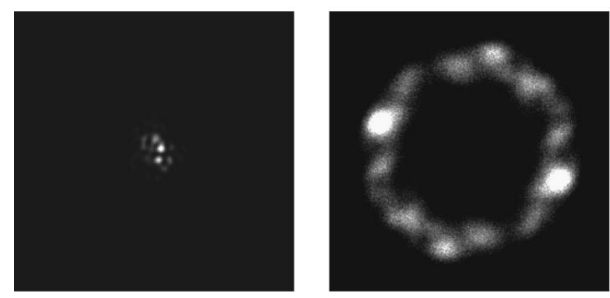

(c)

FIG. 5. Near-field (left) and far-field (right) patterns obtained for $\delta q_{0} / q_{0}=0.05$ (corresponding to a pump beam waist $w_{0}=920 \mu \mathrm{m}$ in the LBO case) (a), $\delta q_{0} / q_{0}=0.1 \quad\left(w_{0}=460 \mu \mathrm{m}\right) \quad(\mathrm{b})$, and $\delta q_{0} / q_{0}=0.3\left(w_{0}=150 \mu \mathrm{m}\right)(\mathrm{c}), \sigma_{p} l_{c}=4$ and $\tau_{0}=1.5 \mathrm{ps}$.

width $\delta q_{0}=2 / w_{0}$, both in the near-field and in the far-field planes. In the near field, the intensity follows the Gaussian profile of the pump and displays a noisy spot pattern with a characteristic wavelength $\pi / q_{R}$. In the far field, the intensity peaks (white spots in the figures) always appear in symmetrical pairs as in the plane-wave pump case illustrated in Fig. 3(b). However, these become broader and broader as the pump beam waist $w_{0}$ beam is reduced. Their size in the observation plane $\pi^{\prime}$ is on the order of the resolution length imposed by the finite transverse size of the pump beam waist, i.e., $x_{\text {diff }}=\lambda f / 2 \pi \delta q_{0}$. The following quantitative evaluations of the amount of correlation included consider only one transverse dimension in space, since a full two-dimensional (2D) calculation (two transverse dimensions + time) would have required an exceedingly long CPU calculation time for our computer.

Some insight can be gained by looking at the n.o. photonnumber correlation $\left\langle: \delta N(\vec{x}) \delta N\left(\vec{x}^{\prime}\right):\right\rangle_{\pi^{\prime}}$ between two pixels centered at $\vec{x}$ and $\vec{x}^{\prime}$, defined by an expression similar to Eq. (30) which refers to the type-II phase-matching configuration. The vectors $\vec{x}$ and $\vec{x}^{\prime}$ denote here the positions of the two pixel detectors in the transverse plane (typically two pixels of a CCD camera), their size being determined in the simulation by the spatial step of the numerical grid. In the degenerate case considered here the indices $i, j$ are dropped since the signal and idler fields are not distinguishable. Integration in time is performed over an interval $T_{d}$ which is taken larger than the pump pulse time, $\tau_{0}=1.5 \mathrm{ps}$, so that all the down-converted photons generated by a single pump 

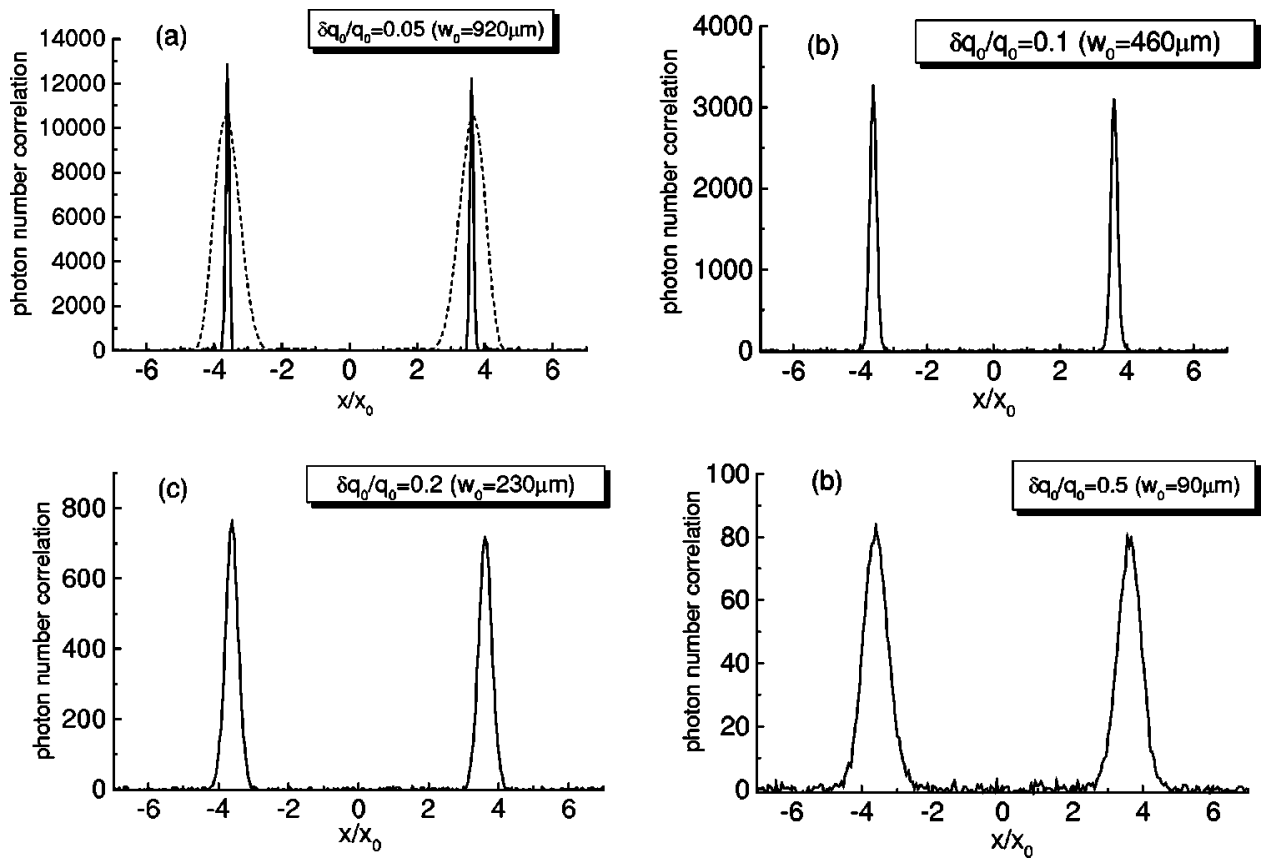

FIG. 6. Far-field correlations: $\left\langle: \delta N(\vec{x}) \delta N\left(\vec{x}^{\prime}\right):\right\rangle_{\pi^{\prime}}$ is plotted as a function of $x$, for increasing values of the ratio $\delta q_{0} / q_{0}$. In (a) the mean photon-number distribution profile is also shown (dotted line). $x^{\prime}=3.6 x_{0}$ is kept fixed in the region of maximum gain. The other parameters are the same as in Fig. 5.

pulse are collected by the two detectors. Its 1D (one transverse dimension + time) numerical evaluation is plotted in Fig. 6 as a function of $x$, keeping $x^{\prime}$ fixed at $x^{\prime}$ $=(\lambda f / 2 \pi) q_{R}$, where the gain is maximum, and for different values of the ratio $\delta q_{0} / q_{0}$. The $x$ coordinate is normalized to $x_{0}=\lambda f / 2 \pi q_{0}$, the spatial scale of the photon-number distribution in the far-field plane. According to these simulations, the widths of the two peaks are on the order of $x_{\text {diff }}$, the resolution length imposed by the pump beam transverse dimensions. The correlation peak at $x=-x^{\prime}$, left in the plots of Fig. 6, is always higher than the correlation peak on the right at $x=x^{\prime}$. In a similar way as was shown in Sec. V [see Eqs. (29) and (37)], this particular behavior of the n.o. correlation function indicates the possibility that the fluctuations of $N_{-}=N_{1}(\vec{x})-N_{2}\left(\vec{x}^{\prime}\right)$ vanish when measured from two symmetrical pixels (i.e., taking $\vec{x}=-\vec{x}^{\prime}$ ). Indeed, for two disconnected pixels we have

$$
\begin{aligned}
\left\langle\left(\delta N_{-}\right)^{2}\right\rangle= & \left\langle N_{+}\right\rangle+\left\langle:[\delta N(\vec{x})]^{2}:\right\rangle+\left\langle:\left[\delta N\left(\vec{x}^{\prime}\right)\right]^{2}:\right\rangle \\
& -2\left\langle\delta N(\vec{x}) \delta N\left(\vec{x}^{\prime}\right)\right\rangle,
\end{aligned}
$$

where $\left\langle N_{+}\right\rangle \equiv\left\langle N(\vec{x})+N\left(\vec{x}^{\prime}\right)\right\rangle$ represents the shot noise for $N_{-}$; therefore, since $\left\langle\left(\delta N_{-}\right)^{2}\right\rangle$ is always a non-negative quantity, the following inequality holds:

$$
2\left\langle\delta N(\vec{x}) \delta N\left(\vec{x}^{\prime}\right)\right\rangle-\left\langle:[\delta N(\vec{x})]^{2}:\right\rangle-\left\langle:\left[\delta N\left(\vec{x}^{\prime}\right)\right]^{2}:\right\rangle \leqslant\left\langle N_{+}\right\rangle .
$$

In particular, when the equality sign holds in Eq. (46), the maximum amount of correlation between points $\vec{x}$ and $\vec{x}^{\prime}$ is achieved, implying thus a complete suppression of the noise of $N_{-}$.
However, the amount of noise in $N_{-}$depends on the actual size of the detectors used to probe the correlation. We have evaluated numerically the variance of $N_{-}$for two symmetric detection areas, by varying the size of the detectors as could be obtained in practice by grouping several pixels of a CCD. The results are shown in Fig. 7, where the different lines correspond to different values of the ratio $\delta q_{0} / q_{0}$, that is, to different values of the pump beam waist. Fluctuations are well below shot noise when the detector size $d$ is larger than $x_{\text {diff }}$, that is, for $d / x_{0}>\delta q_{0} / q_{0}$, which in turn implies that the detection size must be larger than the width of the correlation peaks. These simulations show that the localized character of the correlation predicted by the plane-wave pump theory is well preserved as long as the pump beam waist is not too small. Only in the worst case considered, with $\delta q_{0} / q_{0}=0.5$, the noise reduction factor $\left\langle\left(N_{-}\right)^{2}\right\rangle_{\pi^{\prime}} /\left\langle N_{+}\right\rangle_{\pi^{\prime}}$ is never close to zero unless the detec-

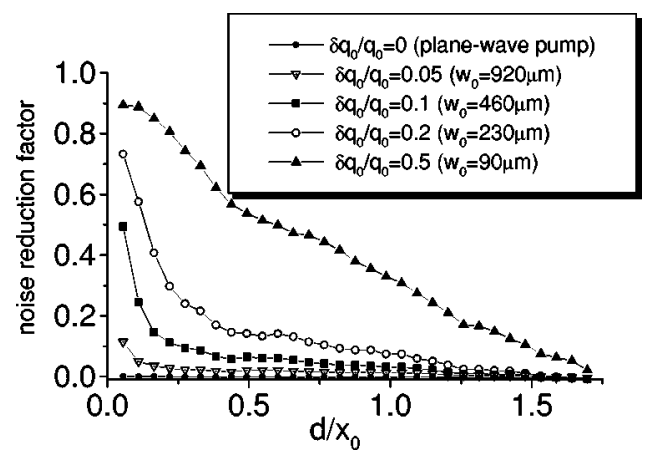

FIG. 7. Far-field correlation in type I: $\left\langle\left(\delta N_{-}\right)^{2}\right\rangle_{\pi^{\prime}} /\left\langle N_{+}\right\rangle_{\pi^{\prime}}$ is plotted as a function of the detector size for different values of $\delta q_{0} / q_{0}$. The other parameters are the same as in the Fig. 6 . 


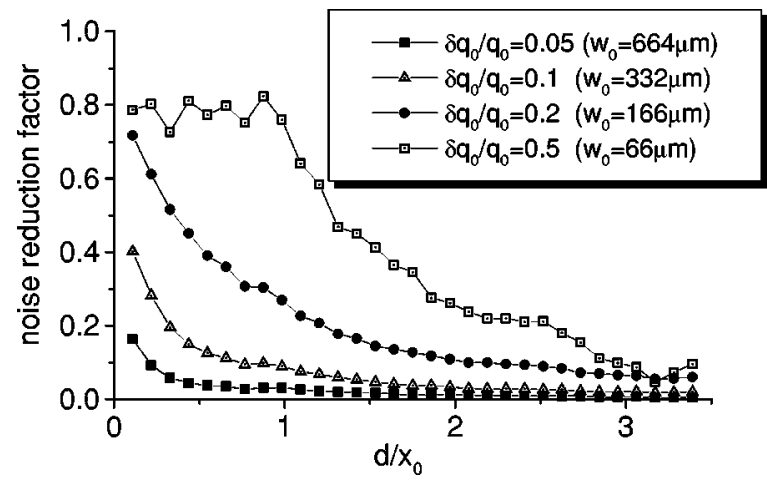

FIG. 8. Far-field correlation: the ratio $\left\langle\left(\delta N_{-}\right)^{2}\right\rangle_{\pi^{\prime}} /\left\langle N_{+}\right\rangle_{\pi^{\prime}}$ is plotted as a function of the detector size $d$ for increasing value of the ratio $\delta q_{0} / q_{0}$. The parametric gain is $\sigma_{p} l_{c}=4$. The negative value of the collinear phase mismatch, $\Delta_{0} l_{c}=-q_{C}^{2} / q_{0}^{2}=-74.4$, is such that the radii of the rings $q_{R}$ vanish.

tors cover the whole width of the ring pattern. It should be noted that a further increase of the ratio $\delta q_{0} / q_{0}$ would lead to single-mode emission: the inverse of this ratio provides indeed an estimation of the number of spatial modes that are efficiently amplified when the field is observed at a fixed temporal frequency.

\section{B. Far-field correlation in type-II crystals}

For the case of a type-II phase-matching configuration, we shall consider explicitly the system described in Ref. [26]: a 1.5-ps high-intensity laser pulse is injected in a 4-mm-long beta barium borate (BBO) crystal cut for type-II phase matching. In the example we consider the pump is oriented at an angle close to $48.2^{\circ}$ with respect to the crystal axis and PDC is observed around the degenerate wavelength $\lambda_{1}=\lambda_{2}=704 \mathrm{~nm}$ with a $10-\mathrm{nm}$ interference filter. For the chosen parameters, the radii of the two rings $q_{R}$ vanish, which is the situation illustrated in Fig. 2(c). As for the type-I configuration, we investigated the momentum correlation that can be observed in the far-field plane $\pi^{\prime}$ by considering two symmetrical detection areas. The variance of $N_{-}$normalized to shot noise is plotted in Fig. 8 as a function of the detector size $d$ and for different values of the pump beam waist. The result is similar to that obtained for the type-I crystal configuration illustrated in Fig. 7: fluctuations are well below shot noise only if $d$ is larger than the characteristic resolution length of the system $x_{\text {diff }}=\lambda f / 2 \pi \delta q_{0}$. We note that the number of temporal modes that are amplified by the crystal is much lower than in the degenerate type-I configuration, since in this case temporal walk-off between the signal and idler fields reduces drastically the emission bandwidth $\Omega_{0}$. Indeed, for the picosecond pump pulse we considered here $\delta \omega_{0} / \Omega_{0}$ is in the order of the unity for the type-II configuration, against the 0.02 value found for the type-I LBO crystal at degeneracy, for which temporal walkoff is not present. The numerical simulations show that this feature of type-II phase matching does not affect spatial correlations, but simply lowers the number of generated photon pairs per pulse.

\section{Near-field correlation in type-II crystals}

A main advantage of the type-II configuration lies in the fact that the signal and idler fields have different polarizations and can therefore be manipulated more easily. In particular, it is possible to measure their mutual correlation in the near field after they have been physically separated by a polarizing beam splitter, as shown schematically in Fig. 4. The lenses $L$ and $L^{\prime}$ shown in the figure simply perform the $2 f-2 f$ imaging of the "near-field plane" $\pi$ onto the two detection planes. For the moment, we only assume that plane $\pi$ is located at some coordinate $z$ inside the crystal. The S-I field self- and cross-correlation functions, which in the plane-wave pump limit have expressions (38a) and (38b), display pronounced peaks for $\vec{x}^{\prime}=\vec{x}$. In particular, the crosscorrelation peak of $\Gamma_{12}\left(\vec{x}, \vec{x}^{\prime}, \Omega\right)$ describes the position entanglement of the $\mathrm{S}+\mathrm{I}$ photons, which are generated in pairs in the same region of the crystal. The width of the peaks is on the order of the coherence length $x_{c o h}=1 / q_{0}$ defined in Eq. (39). It reflects the spread out of the generated photons due to diffraction, which increases proportionally to the square root of the propagation distance. In addition, depending on the phase-matching conditions, twin photons can be emitted noncollinearly with an aperture angle on the order of $\alpha_{R}=2 q_{R} / \bar{k}, q_{R}$ being the radius of the rings in Fourier space given by Eq. (26). This introduces a further indeterminacy on the order of $\alpha_{R} l_{c} / 2=q_{R} / q_{0} x_{c o h}$ in the relative positions of the twin photons measured in the near field. We expect therefore that the two macroscopic fields display identical fluctuations when observed from the same region of the near-field plane, provided that the detection areas $R_{1}$ and $R_{2}$ are larger than the indeterminacy introduced by these propagation effects. However, for crystal lengths on the order of a few millimeters this indeterminacy can be as large as several tens to hundreds of micrometers, so that a substantial portion of the two beams must be intercepted in order to measure significant correlation in the quantum domain. We shall see with a specific example that this difficulty can be at least partially overcome.

The role of spatial walk-off is more subtle to determine. As pointed out in Ref. [31], the Poynting vectors of the phase-matched modes, which determine the photon fluxes of the signal and idler beams, generate two cones that have the same axis inside the crystal. To verify this point explicitly, we consider the Poynting vectors associated with two particular modes of the signal and idler fields at $\Omega=0$ with transverse wave vectors $\vec{q}_{1}$ and $\vec{q}_{2}$. Within the paraxial approximation their directions are determined by the (twodimensional) angles

$$
\vec{\alpha}_{1}=\frac{\vec{q}_{1}}{k_{1}}, \quad \vec{\alpha}_{2}=\frac{\vec{q}_{2}}{k_{2}}+\vec{\rho}_{2},
$$

where $\vec{\rho}_{2} \equiv\left(0,-\rho_{2}\right)$ indicates the walk-off direction of the idler field (the minus sign is due to the fact that in the chosen reference frame the walk-off is oriented opposite to the $y$ axis, $\rho_{0}$ and $\rho_{2}$ being assumed to be positive). For the modes propagating along the axis of the signal and idler cones, we 


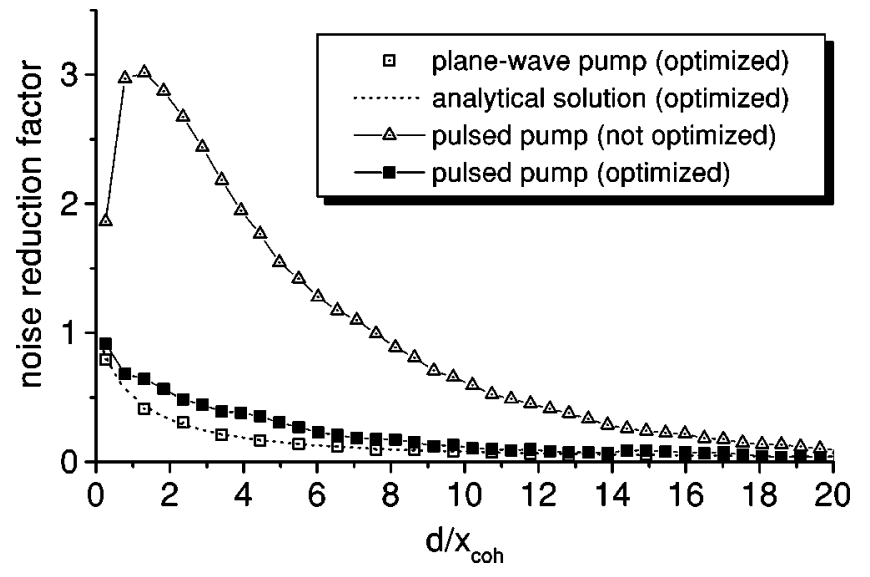

FIG. 9. Near-field correlation: the ratio $\left\langle\left(\delta N_{-}\right)^{2}\right\rangle_{\pi} /\left\langle N_{+}\right\rangle_{\pi}$ is plotted as a function of the detector size. The parameters of the pulsed Gaussian pump are $w_{0}=332 \mu \mathrm{m}\left(\delta q_{0} / q_{0}=0.1\right)$ and $\tau_{0}$ $=1.5 \mathrm{ps}\left(\delta \omega_{0} / \Omega_{0}=1.14\right)$; the gain is $\sigma_{p} l_{c}=3$ and $q_{R}=0$. The simulations performed applying diffraction and walk-off compensation (squares) are well below the one performed without optimization (white triangle). The dashed line corresponds to the analytical solution obtained in the PWPA, given by Eqs. (29) and (41).

have $\vec{q}_{1}=\left(0,-q_{C}\right)$ and $\vec{q}_{2}=\left(0, q_{C}\right)$ with $q_{C}=\frac{1}{2} \bar{k} \rho_{2}$, from which we see that the corresponding Poynting vectors are collinear with $\vec{\alpha}_{1}=\vec{\alpha}_{2}=\left(0,-k_{2} /\left(k_{1}+k_{2}\right) \rho_{2}\right)$. As a result, if the near field is measured directly on the crystal output face $z=l_{c}$, walk-off does not contribute to the indeterminacy in the relative position of the twin photons. It should be stressed, however, that free propagation beyond the crystal occurs at angles which are simply proportional to the transverse wave vector of the phase-matched modes, since in free space the Poynting vector and the $k$-vector directions again coincide. Outside the crystal, the signal and idler emission cones are therefore oriented along different directions with an aperture angle $\alpha_{C}=2 q_{R} / k^{(v)}=\bar{k} / k^{(v)} \rho_{2}$, as illustrated schematically in Fig. 1(a), and give rise to separate rings in the far field $\left(k^{(v)}=2 \pi / \lambda_{1}=2 \pi / \lambda_{2}\right.$ denote here the wave number of the S-I fields in free space at the carrier frequencies). In the simulation illustrated in Fig. 9, we consider the type-II BBO crystal in the same conditions described in Sec. VI B. The near-field coherence length is $x_{c o h}=16.6 \mu \mathrm{m}$ and $q_{R}=0$. The plot displays the noise reduction factor $\left\langle\left(N_{-}\right)^{2}\right\rangle_{\pi} /\left\langle N_{+}\right\rangle_{\pi}$, evaluated numerically as a function of the 1D detector size $d$. If the near-field observation plane $\pi$ coincides with the output face of the crystal at $z=l_{c}$ (white circle), we see that the fluctuations are significantly reduced only when $d$ is about 15 times larger than $x_{c o h}$. The improved result (black squares) has been obtained by imaging onto the detection planes a plane inside the crystal at $z=l_{c}$ $-\Delta z$ rather than the crystal output face. Furthermore, the arrays of pixel detectors in the signal and idler arms are shifted with respect to each other by a distance $\Delta y$ in the transverse direction of walk-off. Notice that this is a $1 \mathrm{D}$ simulation, and the quantities $\Delta y$ and $\Delta z$ correspond to the quantities $\vec{x}_{1}-\vec{x}_{2}$ and $l_{c}-z$, respectively, which appears in definition (42b) of the function $H_{12}\left(\vec{q}, \vec{q}^{\prime}\right) . \Delta z$ and $\Delta y$ are

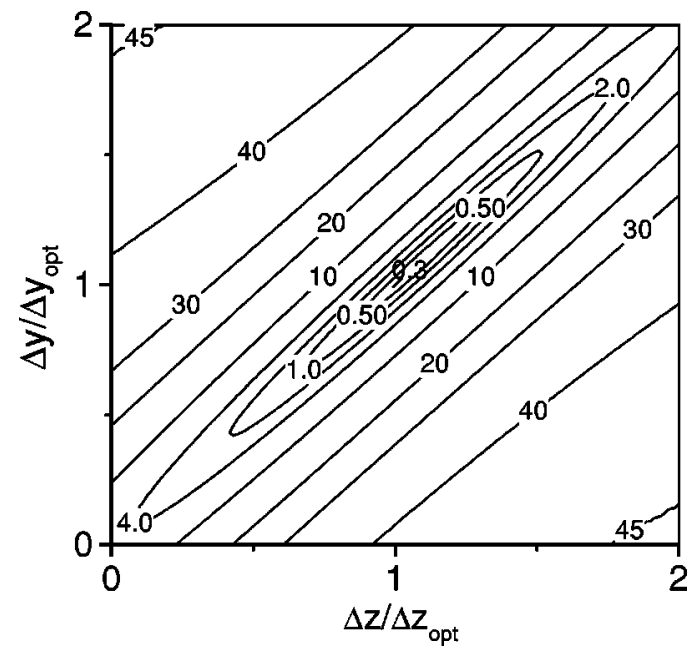

FIG. 10. Near-field correlation: contour plot of the ratio $\left\langle\left(\delta N_{-}\right)^{2}\right\rangle_{\pi} /\left\langle N_{+}\right\rangle_{\pi}$ in the $(\Delta z, \Delta y)$ plane calculated according to the PWPA theory. The minimum in the center corresponds to the values given by Eqs. (48).

chosen in order to minimize the dependence on $\vec{q}$ and $\vec{q}^{\prime}$ of the phase of the integrand in Eq. (41b), maximizing in this way the photon-number cross correlation $\left\langle\delta N_{1} \delta N_{2}\right\rangle$. This is achieved by taking

$$
\begin{gathered}
\Delta z_{o p t}=\frac{\tanh \sigma_{p} l_{c}}{2 \sigma_{p} l_{c}} \frac{n_{1}+n_{2}}{2 n_{1} n_{2}} l_{c}, \\
\Delta y_{o p t}=\frac{\tanh \sigma_{p} l_{c}}{2 \sigma_{p} l_{c}} \rho_{2} l_{c} .
\end{gathered}
$$

Indeed, with these values of $\Delta y$ and $\Delta z$ the phase factor of the function $H_{12}\left(\vec{q}, \vec{q}^{\prime}\right)$ defined by Eq. (42b) nearly cancels the phase of the gain function product appearing in the rhs of Eq. (41b), as can be verified by using the approximate expression

$$
\begin{aligned}
\arg & {\left[U_{1}(\vec{q}, \Omega) V_{2}(-\vec{q},-\Omega) U_{1}^{*}\left(\vec{q}^{\prime}, \Omega\right) V_{2}^{*}\left(-\vec{q}^{\prime},-\Omega\right)\right] } \\
& \approx \frac{\tanh \sigma_{p} l_{c}}{2 \sigma_{p} l_{c}}\left[\Delta(\vec{q}, \Omega)-\Delta\left(\vec{q}^{\prime}, \Omega\right)\right] l_{c}, \\
& =-\frac{\tanh \sigma_{p} l_{c}}{2 \sigma_{p} l_{c}}\left[\rho_{2}\left(\vec{q}_{y}-\vec{q}_{y}^{\prime}\right)+\frac{q^{2}-q^{\prime 2}}{q_{0}}\right]
\end{aligned}
$$

which holds in the high-gain region of the spatial frequency plane. These shifts of the detection S-I planes are necessary in order to minimize the effects of both diffraction and spatial walk-off.

The plot of Fig. 10 displays the variance of $N_{-}$normalized to shot noise in the $(\Delta z, \Delta y)$ plane, as calculated from Eqs. (41). In this example, $d$ is only twice the coherence length, $x_{c o h}=16.6 \mu \mathrm{m}$, while $\Delta y_{o p t}=47.5 \mu \mathrm{m}$ and $\Delta z_{o p t}$ $=407 \mu \mathrm{m}$ (with $\sigma_{p} l_{c}=3$ ). The fluctuations are well above shot noise everywhere except in the narrow diagonal region around the point $\left(\Delta z_{o p t}, \Delta y_{o p t}\right)$, where $\left\langle\left(\delta N_{-}\right)^{2}\right\rangle_{\pi} /\left\langle N_{+}\right\rangle_{\pi}$ $=0.3$. Outside this region, the self-correlation becomes 
much larger than the cross correlation and the variance of $N_{-}$rapidly exceeds the shot-noise level. This means that for such small detectors a highly precise imaging of the near field determined by Eqs. (48) is therefore necessary in order to observe some noise reduction effect.

Although the previous results rely on a detailed description of the relative phases of the signal and idler fields, we can give them a more intuitive explanation based on the particle picture.

(1) In the low-gain regime $\left(\sigma_{p} l_{c} \ll 1\right)$, photon pairs are generated uniformly along the crystal. In this case the choice $\Delta z \approx l_{c} / 2$ lowers the effect of diffraction and noncollinear propagation, since the mean propagation distance that photon pairs must undergo to reach the imaging plane $\pi$ from the point in which they are created is minimized. The factor depending on the refractive indices takes into account that photons propagate in a dense medium rather than in free space.

(2) On the other hand, the fields imaged from a generic plane inside the crystal lying at distance $\Delta z$ from the output face can be obtained by a virtual free space back-propagation from plane $z=l_{c}$ to plane $z=l_{c}-\Delta z$. As already mentioned, free space propagation leads to an angular divergence of the signal and idler beams with a mean aperture angle equal to $\alpha_{C} \approx \rho_{2}$ along the walk-off direction. The signal and idler photons are therefore pulled apart a distance $\Delta y=\alpha_{C} \Delta z$ $\approx \rho_{2} \Delta z$ when observed in plane $z=l_{c}-\Delta z$. In order to compensate this effect the two detection areas must be separated by the same distance along the the $y$ axis. This explains why the value of $\Delta y$ for which the fluctuations are minimized in a given imaging plane is proportional to $\Delta z$, as appears from the cigar shaped region of low fluctuations along the diagonal direction illustrated in the contour plot of Fig. 10. In particular, for $\Delta z=\Delta z_{\text {opt }}$ walk-off is compensated by taking $\Delta y=\alpha_{C} \Delta z_{\text {opt }}=\rho_{2} l_{c} / 2$ and we obtain thereby the shifts given by Eqs. (48a) and (48b) for the limit $\sigma_{p} l_{c} \ll 1$.

(3) The factor depending on the gain parameter in Eq. (48a), which decreases as $\sigma_{p} l_{c}$ increases, can be understood by noting that in a high-gain regime most of the photon pairs are generated in the last part of the crystal, because of a cascading effect. Hence in order to minimize the propagation distance from the point where they are created to the plane $\pi$, this plane should be taken closer and closer to the crystal exit face as the gain is increased.

Thanks to this procedure, the noise in $N_{-}$is considerably lowered with respect to measurements performed at plane $z$ $=l_{c}$ with aligned detection areas (i.e., with $\Delta z=\Delta y=0$ ). Although values (48a) and(48b) have been evaluated within the plane-wave pump approximation, the numerical simulations demonstrate that the procedure works well even when the pump has a finite size. It should also be emphasized that both shifts (48a) and (48b) proved to be equally necessary in order to obtain this improvement.

\section{CONCLUSIONS}

The results of this paper demonstrate that PDC is able to display spatial correlation effects at the level of quantum fluctuations even in a regime of high gain, i.e., when the down-converted photons form macroscopic fields. The quantum origin of the S-I correlations, which lie in the position and momentum entanglement of the twin photons building up the two beams, can be best demonstrated in a type-II phase-matching configuration where both near-field and farfield measurements can be implemented. We showed numerically that spatial far-field correlations of quantum origin are observable when the pump beam waist is in the millimeter range and the detection areas are larger than the resolution area of the system. Near-field correlations seem more difficult to observe experimentally since propagation tends to destroy the position entanglement of the generated photon pairs. We proposed a detection scheme that allows to optimize their measurement by compensating the detrimental effect of diffraction. These results are strongly related to a recent paper of ours [19], which discusses the topic of entangled imaging and extends this technique to the macroscopic domain. The simultaneous presence of spatial entanglement in both the near and the far field plays a crucial role in the analysis of Ref. [19]. In this paper we provide a more quantitative analysis of the level of quantum correlation which is present in the far field on the one hand and in the near field on the other.

\section{ACKNOWLEDGMENTS}

The authors thank Ottavia Jedrkiewicz, Paolo Di Trapani, Yunkun Jiang, and Eric Lantz for helpful comments and discussions. This work was supported by the European FET Project QUANTIM (Quantum Imaging) and by INTAS Project No. 01-2097. M.B. acknowledges financial support from the Danish Technical Research Council (STVF).

\section{APPENDIX A: INPUT-OUTPUT FORMALISM}

The finite pump pulse bandwidth in space and in time generates coupling between all modes of the S-I field, deteriorating thereby the perfect correlation between the $(\vec{q}, \Omega)_{1}$ and $(-\vec{q},-\Omega)_{2}$ modes. In this section we generalize the input-output formalism of the PWPA to the finite pump case. Input-output transformations (14) are replaced by the more general linear transformation:

$$
\begin{aligned}
a_{1}(z, \vec{q}, \Omega)= & \int d \vec{q}^{\prime} \int d \Omega^{\prime}\left[\mathcal{U}_{1}\left(z ; \vec{q}, \Omega ; \vec{q}^{\prime} \Omega^{\prime}\right)\right. \\
& \times a_{1}^{i n}\left(\vec{q}+\vec{q}^{\prime}, \Omega+\Omega^{\prime}\right)+\mathcal{V}_{1}\left(z ; \vec{q}, \Omega ; \vec{q}^{\prime}, \Omega^{\prime}\right) \\
& \left.\times a_{2}^{i n \dagger}\left(-\vec{q}+\vec{q}^{\prime},-\Omega+\Omega^{\prime}\right)\right], \\
a_{2}(z, \vec{q}, \Omega)= & \int d \vec{q}^{\prime} \int d \Omega^{\prime}\left[\mathcal{V}_{2}\left(z ; \vec{q}, \Omega ; \vec{q}^{\prime}, \Omega^{\prime}\right)\right. \\
& \times a_{1}^{i n \dagger}\left(-\vec{q}+\vec{q}^{\prime},-\Omega+\Omega^{\prime}\right) \\
& \left.+\mathcal{U}_{2}\left(z ; \vec{q}, \Omega ; \vec{q}^{\prime} \Omega^{\prime}\right) a_{2}^{i n}\left(\vec{q}+\vec{q}^{\prime}, \Omega+\Omega^{\prime}\right)\right],
\end{aligned}
$$

which express the fields in a generic plane $z$ inside the crystal in the form of a convolution integral with the input field 
Fourier modes $a_{j}^{i n}(\vec{q}, \Omega)=a_{j}(z=0, \vec{q}, \Omega), j=1,2$. From Eqs. (7), we can obtain a fully equivalent set of equations for the propagation kernels:

$$
\begin{aligned}
& \frac{\partial \mathcal{U}_{1}\left(z ; \vec{q}, \Omega ; \vec{q}^{\prime}, \Omega^{\prime}\right)}{\partial z} \\
&= i \delta_{1}(\vec{q}, \Omega) \mathcal{U}_{1}\left(z ; \vec{q}, \Omega ; \vec{q}^{\prime}, \Omega^{\prime}\right)+\sigma e^{-i \Delta_{0} z} \\
& \times \int \frac{d \vec{q}^{\prime \prime}}{2 \pi} \int \frac{d \Omega^{\prime \prime}}{\sqrt{2 \pi}} A_{0}\left(z, \vec{q}^{\prime \prime}, \Omega^{\prime \prime}\right) \mathcal{V}_{2}^{*}\left(z ; \vec{q}^{\prime \prime}-\vec{q}, \Omega^{\prime \prime}\right. \\
&\left.-\Omega ; \vec{q}^{\prime}+\vec{q}^{\prime \prime}, \Omega^{\prime}+\Omega^{\prime \prime}\right), \\
& \frac{\partial \mathcal{V}_{2}\left(z ; \vec{q}, \Omega, \vec{q}^{\prime}, \Omega^{\prime}\right)}{\partial z} i \delta_{2}(\vec{q}, \Omega) \mathcal{V}_{2}\left(z ; \vec{q}, \Omega, \vec{q}^{\prime}, \Omega^{\prime}\right)+\sigma e^{-i \Delta_{0} z} \\
& \quad \times \int \frac{d \vec{q}^{\prime \prime}}{2 \pi} \int \frac{d \Omega^{\prime \prime}}{\sqrt{2 \pi}} A_{0}\left(z, \vec{q}^{\prime \prime}, \Omega^{\prime \prime}\right) \\
& \quad \times \mathcal{U}_{1}^{*}\left(z ; \vec{q}^{\prime \prime}-\vec{q}, \Omega^{\prime \prime}-\Omega ; \vec{q}^{\prime}+\vec{q}^{\prime \prime}, \Omega^{\prime}+\Omega^{\prime \prime}\right) .
\end{aligned}
$$

The equations for the remaining kernels $\mathcal{V}_{1}$ and $\mathcal{U}_{2}$ can be obtained by interchanging indices 1 and 2 , and the following initial conditions must be fulfilled:

$$
\begin{aligned}
& \mathcal{U}_{j}\left(z=0 ; \vec{q}, \Omega ; \vec{q}^{\prime}, \Omega^{\prime}\right)=\delta\left(\vec{q}^{\prime}\right) \delta\left(\Omega^{\prime}\right), \\
& \mathcal{V}_{j}\left(z=0 ; \vec{q}, \Omega ; \vec{q}^{\prime}, \Omega^{\prime}\right)=0 \quad(j=1,2) .
\end{aligned}
$$

It can be shown that the solutions of this set of equations satisfy the relations

$$
\begin{aligned}
\int d \vec{q}^{\prime \prime} \int & d \Omega^{\prime \prime}\left[\mathcal{U}_{1}\left(z ; \vec{q}, \Omega ; \vec{q}^{\prime \prime}-\vec{q}, \Omega^{\prime \prime}-\Omega\right)\right. \\
& \times \mathcal{U}_{1}^{*}\left(z ; \vec{q}, \Omega ; \vec{q}^{\prime \prime}-\vec{q}^{\prime}, \Omega^{\prime \prime}-\Omega^{\prime}\right)-\mathcal{V}_{1}\left(z ; \vec{q}, \Omega ; \vec{q}^{\prime \prime}\right. \\
+ & \left.\left.\vec{q}, \Omega^{\prime \prime}+\Omega\right) \mathcal{V}_{1}^{*}\left(z ; \vec{q}, \Omega ; \vec{q}^{\prime \prime}+\vec{q}^{\prime}, \Omega^{\prime \prime}+\Omega^{\prime}\right)\right] \\
= & \delta\left(\vec{q}^{\prime}-\vec{q}\right) \delta\left(\Omega-\Omega^{\prime}\right), \\
\int \quad d & \vec{q}^{\prime \prime} \int d \Omega^{\prime \prime} \mathcal{U}_{1}\left(z ; \vec{q}, \Omega ; \vec{q}^{\prime \prime}-\vec{q}, \Omega^{\prime \prime}-\Omega\right) \\
\quad & \times \mathcal{V}_{2}\left(z ; \vec{q}, \Omega ; \vec{q}^{\prime \prime}+\vec{q}^{\prime}, \Omega^{\prime \prime}+\Omega^{\prime}\right) \\
= & \int d \vec{q}^{\prime \prime} \int d \Omega \Omega^{\prime \prime} \mathcal{V}_{1}\left(z ; \vec{q}, \Omega ; \vec{q}^{\prime \prime}+\vec{q}, \Omega^{\prime \prime}+\Omega\right) \\
& \times \mathcal{U}_{2}\left(z ; \vec{q}, \Omega ; \vec{q}^{\prime \prime}-\vec{q}^{\prime}, \Omega \Omega^{\prime \prime}-\Omega^{\prime}\right),
\end{aligned}
$$

which generalize the unitarity conditions (17) beyond the case of plane wave and cw pump.

The normally ordered photon-number correlation function in a generic transverse plane $z$ can be written as

$$
\begin{aligned}
G_{i j}^{(z)} & \left(\vec{x}, t, \vec{x}^{\prime}, t^{\prime}\right) \\
= & \left\langle a_{i}^{\dagger}(z, \vec{x}, t) a_{j}^{\dagger}\left(z, \vec{x}^{\prime}, t^{\prime}\right) a_{j}\left(z, \vec{x}^{\prime}, t^{\prime}\right) a_{i}(z, \vec{x}, t)\right\rangle \\
& -\left\langle a_{i}^{\dagger}(z, \vec{x}, t) a_{i}(z, \vec{x}, t)\right\rangle\left\langle a_{j}^{\dagger}\left(z, \vec{x}^{\prime}, t^{\prime}\right) a_{j}\left(z, \vec{x}^{\prime}, t^{\prime}\right)\right\rangle \quad(\mathrm{A} 5 \mathrm{a}) \\
& =\left|\left\langle a_{i}^{\dagger}(z, \vec{x}, t) a_{j}\left(z, \vec{x}^{\prime}, t^{\prime}\right)\right\rangle\right|^{2}+\left|\left\langle a_{i}(z, \vec{x}, t) a_{j}\left(z, \vec{x}^{\prime}, t^{\prime}\right)\right\rangle\right|^{2} .
\end{aligned}
$$

In the last identity we made use of the general property characterizing fields with Gaussian statistics, which allows to write the fourth-order field correlations as a sum of the products of the second-order correlation functions (see, e.g., Ref. [32]). We now consider explicitly the transformations relating the S-I fields in the planes where detection is performed, to those on the crystal output face, $a_{j}^{\text {out }}(\vec{q}, t)$ :

$$
\begin{aligned}
a_{j}(z, \vec{x}, t) & =\int d \vec{q} h_{j}(\vec{x}, \vec{q}) a_{j}^{\text {out }}(\vec{q}, t) \\
& =\int d \vec{x}^{\prime} h_{j}\left(\vec{x}, \vec{x}^{\prime}\right) a_{j}^{\text {out }}\left(\vec{x}^{\prime}, t\right) \quad(j=1,2),
\end{aligned}
$$

with

$$
h_{j}(\vec{x}, \vec{q}) \equiv \int \frac{d \vec{x}^{\prime}}{2 \pi} e^{i \vec{q} \cdot \vec{x}^{\prime}} h_{j}\left(\vec{x}, \vec{x}^{\prime}\right) .
$$

Propagation outside the crystal can include several optical devices, such as lenses and polarizing beam splitters, and can take different paths for the signal and idler beams (see scheme of Fig. 4). We shall assume, however, that it occurs without losses and this latter condition implies that the Fresnel kernels $h_{j}(\vec{x}, \vec{q})$ satisfy the relation

$$
\int d \vec{x} h_{j}^{*}(\vec{x}, \vec{q}) h_{j}\left(\vec{x}, \vec{q}^{\prime}\right)=\delta\left(\vec{q}-\vec{q}^{\prime}\right) \quad(j=1,2),
$$

which can be obtained by requiring that the commutation rules (4) are preserved in the transformation. When the detection time $T_{d}$ is much longer than the coherence time $\Omega_{0}^{-1}$, the photon-number self- and cross-correlations measured over two detection areas $R_{1}$ and $R_{2}$, as defined by Eq. (30), can be written as

$$
\begin{aligned}
\left\langle:\left(\delta N_{1}\right)^{2}:\right\rangle_{z}= & \int d \Omega \int d \Omega^{\prime} \int_{R_{1}} d \vec{x} \int_{R_{1}} d \vec{x}^{\prime} \\
& \times \mid \int d \vec{q} \int d \vec{q}^{\prime} h_{1}^{*}(\vec{x}, \vec{q}) h_{1}\left(\vec{x}^{\prime}, \vec{q}^{\prime}\right) \\
& \times\left.\left\langle a_{1}^{\text {out }}(\vec{q}, \Omega) a_{1}^{\text {out }}\left(\vec{q}^{\prime}, \Omega^{\prime}\right)\right\rangle\right|^{2},
\end{aligned}
$$




$$
\begin{aligned}
\left\langle\delta N_{1} \delta N_{2}\right\rangle_{z}= & \int d \Omega \int d \Omega^{\prime} \int_{R_{1}} d \vec{x} \int_{R_{2}} d \vec{x}^{\prime} \\
& \times \mid \int d \vec{q} \int d \vec{q}^{\prime} h_{1}(\vec{x}, \vec{q}) h_{2}\left(\vec{x}^{\prime}, \vec{q}^{\prime}\right) \\
& \times\left.\left\langle a_{1}^{\text {out }}(\vec{q}, \Omega) a_{2}^{\text {out }}\left(\vec{q}^{\prime}, \Omega^{\prime}\right)\right\rangle\right|^{2},
\end{aligned}
$$

where we used relation (A6) in order to express the secondorder field correlations appearing in Eqs. (A5b) in terms of the output field operators $a_{j}^{\text {out }}(\vec{q}, \Omega), j=1,2$. The correlation functions of the output fields can be expressed in terms of the propagation kernels defined by Eq. (A1a) evaluated at plane $z=l_{c}$ as

$$
\begin{aligned}
& \left\langle a_{1}^{\text {out } \dagger}(\vec{q}, \Omega) a_{1}^{\text {out }}\left(\vec{q}^{\prime}, \Omega^{\prime}\right)\right\rangle \\
& =\int d \vec{x} \int d t e^{i\left(\vec{q}-\vec{q}^{\prime}\right) \cdot \vec{x}-i(\Omega-\Omega) t} \mathcal{V}_{1}^{*}\left(l_{c} ; \vec{q}, \Omega ; \vec{x}, t\right) \\
& \quad \times \mathcal{V}_{1}\left(l_{c} ; \vec{q}^{\prime}, \Omega^{\prime} ; \vec{x}, t\right), \\
& \left\langle a_{1}^{\text {out }}(\vec{q}, \Omega) a_{2}^{\text {out }}\left(\vec{q}^{\prime}, \Omega^{\prime}\right)\right\rangle \\
& \quad \int d \vec{x} \int d t e^{i\left(\vec{q}+\vec{q}^{\prime}\right) \cdot \vec{x}-i(\Omega+\Omega) t} \mathcal{U}_{1}\left(l_{c} ; \vec{q}, \Omega ; \vec{x}, t\right) \\
& \quad \times \mathcal{V}_{2}\left(l_{c} ; \vec{q}^{\prime}, \Omega^{\prime} ; \vec{x}, t\right), \\
& \left\langle a_{1}^{\text {out }}(\vec{q}, \Omega) a_{1}^{\text {out }}\left(\vec{q}^{\prime}, \Omega^{\prime}\right)\right\rangle=\left\langle a_{1}^{\text {out } \dagger}(\vec{q}, \Omega) a_{2}^{\text {out }}\left(\vec{q}^{\prime}, \Omega^{\prime}\right)\right\rangle=0,
\end{aligned}
$$

with

$$
\begin{aligned}
\mathcal{U}_{j}(z ; \vec{q}, \Omega ; \vec{x}, t)= & \int \frac{d \vec{q}}{2 \pi} \int \frac{d t}{\sqrt{2 \pi}} e^{i \overrightarrow{q^{\prime}} \cdot \vec{x}-i \Omega^{\prime} t} \\
& \times \mathcal{U}_{j}\left(z ; \vec{q}, \Omega ; \vec{q}^{\prime}, \Omega^{\prime}\right) \quad(j=1,2)
\end{aligned}
$$

A similar definition holds for the functions $\mathcal{V}_{j}(z ; \vec{q}, \Omega ; \vec{x}, t)$, $j=1,2$. In case $R_{1}$ and $R_{2}$ intercept all the photons of the signal and idler fields generated in the down-conversion process, it can be shown that the variance of $N_{-}=N_{1}-N_{2}$ vanishes if condition (A8) is fulfilled (i.e., if free propagation occurs without losses). However, in general such a result does not hold if the two detectors collect photons only from finite portions of the two beams.

\section{APPENDIX B: APPROXIMATE SOLUTION IN THE QUASISTATIONARY REGIME}

In this appendix we derive an approximate analytical solution of the propagation equations (7) assuming the spatial and temporal frequency bandwidths of the pump are small but finite; more precisely we assume that conditions (24) are satisfied.

It is useful to write the propagation equations in the ref- erence frame comoving with the pump field envelope, whose coordinates are related to the original laboratory coordinates through the linear transformation $t^{\prime}=t-k_{0}^{\prime} z, y^{\prime}=y+\rho_{0} z$. In Fourier space this corresponds to multiplying the Fourier components of the S-I field envelopes by $e^{-i\left(k_{0}^{\prime} \Omega+\rho_{0} q_{y}\right) z}$. More precisely we consider the transformation

$$
\begin{gathered}
a_{j}^{\prime}(z, \vec{q}, \Omega)=\exp \left(i\left[\frac{\Delta_{0}}{2}-k_{0}^{\prime} \Omega-\rho_{0} q_{y}\right] z\right) a_{j}(z, \vec{q}, \Omega) \\
(j=1,2),
\end{gathered}
$$

where the constant phase factor $e^{i \Delta_{0} / 2 z}$ has been added in order to eliminate $e^{i \Delta_{0} z}$ from the convolution term in Eqs. (A2). Input-output transformations (A1a) and unitarity conditions (A4) still hold for the transformed kernels

$$
\begin{aligned}
& \mathcal{U}_{j}^{\prime}\left(z ; \vec{q}, \Omega ; \vec{q}^{\prime}, \Omega^{\prime}\right) \\
& =\exp \left(i\left[\frac{\Delta_{0}}{2}-k_{0}^{\prime} \Omega-\rho_{0} q_{y}\right] z\right) \mathcal{U}_{j}\left(z ; \vec{q}, \Omega ; \vec{q}^{\prime}, \Omega^{\prime}\right), \\
& \mathcal{V}_{j}^{\prime}\left(z ; \vec{q}, \Omega ; \vec{q}^{\prime}, \Omega^{\prime}\right) \\
& =\exp \left(i\left[\frac{\Delta_{0}}{2}-k_{0}^{\prime} \Omega-\rho_{0} q_{y}\right] z\right) \mathcal{V}_{j}\left(z ; \vec{q}, \Omega ; \vec{q}^{\prime}, \Omega^{\prime}\right) \\
& (j=1,2),
\end{aligned}
$$

which satisfy the propagation equations

$$
\begin{aligned}
& \frac{\partial \mathcal{U}_{1}^{\prime}\left(z ; \vec{q}, \Omega ; \vec{q}^{\prime}, \Omega^{\prime}\right)}{\partial z} i \delta_{1}^{\prime}(\vec{q}, \Omega) \mathcal{U}_{1}^{\prime}\left(z ; \vec{q}, \Omega ; \vec{q}^{\prime}, \Omega^{\prime}\right)+\int \frac{d \vec{q}^{\prime \prime}}{2 \pi} \int \frac{d \Omega^{\prime \prime}}{\sqrt{2 \pi}} \\
& \quad \times e^{i \delta_{0}^{\prime}\left(\vec{q}^{\prime \prime}, \Omega^{\prime \prime}\right) z} A_{0}\left(z=0, \vec{q}^{\prime \prime}, \Omega^{\prime \prime}\right) \\
& \quad \times \mathcal{V}_{2}^{\prime *}\left(z ; \vec{q}^{\prime \prime}-\vec{q}, \Omega^{\prime \prime}-\Omega ; \vec{q}^{\prime}+\vec{q}^{\prime \prime}, \Omega^{\prime}+\Omega^{\prime \prime}\right), \\
& \frac{\partial \mathcal{V}_{2}^{\prime}\left(z ; \vec{q}, \Omega, \vec{q}^{\prime}, \Omega^{\prime}\right)}{\partial z} \\
&=i \delta_{2}^{\prime}(\vec{q}, \Omega) \mathcal{V}^{\prime}{ }_{2}\left(z ; \vec{q}, \Omega, \vec{q}^{\prime}, \Omega^{\prime}\right)+\int \frac{d \vec{q}^{\prime \prime}}{2 \pi} \int \frac{d \Omega^{\prime \prime}}{\sqrt{2 \pi}} \\
& \quad \times e^{i \delta_{0}^{\prime}\left(\vec{q}^{\prime \prime}, \Omega^{\prime \prime}\right) z} A_{0}\left(z=0, \vec{q}^{\prime \prime}, \Omega^{\prime \prime}\right) \\
& \quad \times \mathcal{V}_{1}^{\prime *}\left(z ; \vec{q}^{\prime \prime}-\vec{q}, \Omega^{\prime \prime}-\Omega^{\prime} ; \vec{q}^{\prime}+\vec{q}^{\prime \prime}, \Omega^{\prime}+\Omega^{\prime \prime}\right) .
\end{aligned}
$$

The explicit form for the propagation of the pump field (9) has been used and we defined the new detuning parameters

$$
\delta_{j}^{\prime}(\vec{q}, \Omega)=\frac{\Delta_{0}}{2}+\delta_{j}(\vec{q}, \Omega)-\rho_{0} q_{y}-k_{0}^{\prime} \Omega \quad(j=1,2),
$$




$$
\delta_{0}^{\prime}(\vec{q}, \Omega)=\frac{1}{2} k_{0}^{\prime \prime} \Omega^{2}-\frac{1}{2 k_{0}} q^{2} .
$$

Remembering the hypothesis that the pump envelope Fourier transform at plane $z=0$ has the Gaussian form (11) with $\delta q_{0} \ll q_{0}$ and $\delta \omega_{0} \ll \Omega_{0}$, we can now apply the following approximations.

(1) The phase term in the convolution integrals can be neglected, since $\delta_{0}^{\prime}(\vec{q}, \Omega) l_{c}$ is at most on the order of $\max \left(\delta q_{0}^{2} / q_{0}^{2}, \delta \omega_{0}^{2} / \Omega_{0}^{2}\right)$ in the region where the Fourier transform of the pump envelope is not negligible.

(2) We expect that the propagation kernels defined by Eq. (A1a) are characterized by the slow variation scale $\left(q_{0}, \Omega_{0}\right)$ in their unprimed arguments, while they are strongly peaked in the origin of the primed variable space $\left(\vec{q}^{\prime}, \Omega^{\prime}\right)$, in which they have a much faster variation scale. This assumption is justified by the form of the solutions of Eqs. (B3) obtained in the PWPA limit. As $\delta q_{0} / q_{0} \rightarrow 0$ and $\delta \omega_{0} / \Omega_{0} \rightarrow 0$, the primed propagation kernels satisfying initial conditions (A3) take indeed the simple form:

$$
\begin{aligned}
& \mathcal{U}_{j}^{\prime}\left(z ; \vec{q}, \Omega ; \vec{q}^{\prime}, \Omega^{\prime}\right) \\
& \quad=\delta\left(\vec{q}^{\prime}\right) \delta\left(\Omega^{\prime}\right) \exp \left(i\left[\frac{\Delta_{0}}{2}-k_{0}^{\prime} \Omega-\rho_{0} q_{y}\right] z\right) U_{j}(z ; \vec{q}, \Omega),
\end{aligned}
$$

$$
\begin{aligned}
& \mathcal{V}_{j}^{\prime}\left(z ; \vec{q}, \Omega ; \vec{q}^{\prime}, \Omega^{\prime}\right) \\
& \quad=\delta\left(\vec{q}^{\prime}\right) \delta\left(\Omega^{\prime}\right) \exp \left(i\left[\frac{\Delta_{0}}{2}-k_{0}^{\prime} \Omega-\rho_{0} q_{y}\right] z\right) V_{j}(z ; \vec{q}, \Omega),
\end{aligned}
$$

where the gain functions $U_{j}(z ; \vec{q}, \Omega)$ and $V_{j}(z ; \vec{q}, \Omega)$ are given by Eqs. (15), with $l_{c}$ being replaced by the $z$ coordinate. This latter hypothesis allows us to neglect the dependence on $\vec{q}^{\prime \prime}$ and $\Omega^{\prime \prime}$ in the first argument of the kernels in the convolution integrals at the rhs of Eqs. (B3a) and (B3b). With these approximations, we obtain a system that can be solved analytically and which acquires its simplest form when written for the kernels Fourier transformed in their primed arguments [see definition (A11)]:

$$
\begin{aligned}
\frac{\partial \mathcal{U}_{1}^{\prime}(\vec{q}, \Omega ; \vec{x}, t)}{\partial z}= & i \delta_{1}^{\prime}(\vec{q}, \Omega) \mathcal{U}_{1}^{\prime}(\vec{q}, \Omega ; \vec{x}, t)+\sigma A_{0}(z=0, \vec{x}, t) \\
& \times \mathcal{V}_{2}^{\prime *}(-\vec{q},-\Omega ;-\vec{x},-t), \\
\frac{\partial \mathcal{V}_{2}^{\prime}(\vec{q}, \Omega, \vec{x}, t)}{\partial z}= & i \delta_{2}^{\prime}(\vec{q}, \Omega) \mathcal{V}_{2}^{\prime}(\vec{q}, \Omega, \vec{x}, t)+\sigma A_{0}(z=0, \vec{x}, t) \\
& \times \mathcal{U}_{1}^{\prime} *(-\vec{q},-\Omega ;-\vec{x},-t),
\end{aligned}
$$

The solution of this system satisfying initial conditions (A3), which now read $\mathcal{U}_{j}^{\prime}(z=0 ; \vec{q}, \Omega ; \vec{x}, t)=1 /(2 \pi)^{3 / 2}, \quad \mathcal{V}_{j}^{\prime}(z$ $=0 ; \vec{q}, \Omega ; \vec{x}, t)=0,(j=1,2)$, are

$$
\begin{aligned}
\mathcal{U}^{\prime}{ }_{1}(z ; \vec{q}, \Omega ; \vec{x}, t)= & \exp \left[i \frac{\delta_{1}^{\prime}(\vec{q}, \Omega)-\delta_{2}^{\prime}(-\vec{q},-\Omega)}{2} z\right] \\
& \times U(z ; \vec{q}, \Omega ; \vec{x}, t) \\
\mathcal{V}^{\prime}{ }_{1}(z ; \vec{q}, \Omega ; \vec{x}, t)= & \exp \left[i \frac{\delta_{1}^{\prime}(\vec{q}, \Omega)-\delta_{2}^{\prime}(-\vec{q},-\Omega)}{2} z\right] \\
& \times V(z ; \vec{q}, \Omega ; \vec{x}, t),
\end{aligned}
$$$$
\mathcal{U}_{2}^{\prime}(z ; \vec{q}, \Omega ; \vec{x}, t)=\exp \left[i \frac{\delta_{2}^{\prime}(\vec{q}, \Omega)-\delta_{1}^{\prime}(-\vec{q},-\Omega)}{2} z\right]
$$$$
\times U(z ;-\vec{q},-\Omega ;-\vec{x},-t),
$$$$
\mathcal{V}_{2}^{\prime}(z ; \vec{q}, \Omega ; \vec{x}, t)=\exp \left[i \frac{\delta_{2}^{\prime}(\vec{q}, \Omega)-\delta_{1}^{\prime}(-\vec{q},-\Omega)}{2} z\right]
$$$$
\times V(z ;-\vec{q},-\Omega ;-\vec{x},-t),
$$

with

$$
\begin{aligned}
U(z ; \vec{q}, \Omega ; \vec{x}, t)= & \frac{1}{(2 \pi)^{3 / 2}}[\cosh \Gamma(\vec{q}, \Omega, \vec{x}, t) z \\
& \left.+i \frac{\Delta(\vec{q}, \Omega)}{2 \Gamma(\vec{q}, \Omega, \vec{x}, t)} \sinh \Gamma(\vec{q}, \Omega, \vec{x}, t) z\right]
\end{aligned}
$$

$$
\begin{aligned}
V(z ; \vec{q}, \Omega ; \vec{x}, t) & =\frac{1}{(2 \pi)^{3 / 2}} \frac{\sigma A_{0}(\vec{x}, t)}{\Gamma(\vec{q}, \Omega, \vec{x}, t)} \sinh \Gamma(\vec{q}, \Omega, \vec{x}, t) z \\
\Gamma(\vec{q}, \Omega ; \vec{x}, t) & =\sqrt{\sigma^{2} A_{0}^{2}(\vec{x}, t)-\frac{\Delta(\vec{q}, \Omega)^{2}}{4}}, \\
\Delta(\vec{q}, \Omega) & =\delta_{1}^{\prime}(\vec{q}, \Omega)+\delta_{2}^{\prime}(-\vec{q},-\Omega) \\
& =\Delta_{0}+\delta_{1}(\vec{q}, \Omega)+\delta_{2}(-\vec{q},-\Omega)
\end{aligned}
$$

Clearly, as $\delta q_{0}, \delta \omega_{0} \rightarrow 0$ these function lose their dependence on the space-time coordinates $(\vec{x}, t)$ and we recover the PWPA solution expressed by Eqs. (15) and (16). In the more general case in which the ratios $\delta q_{0} / q_{0}$ and $\delta q_{0} / q_{0}$ are small but finite, the self-correlation function (A10a) is peaked at $\vec{q}^{\prime}=\vec{q}$, while the cross-correlation function (A10b) is peaked at $\vec{q}^{\prime}=-\vec{q}$, the width of both peaks being on the order of $\delta q_{0}$. This is more clearly seen by considering the special limit in which $l_{c} \rightarrow 0$ and the gain parameter $\sigma A_{p} l_{c}$ remains a finite quantity. In this case the effects of linear propagation, such as diffraction, dispersion and walk-off, become negligible and the propagation kernels (B8) lose their 
dependence on $\vec{q}$ and $\Omega$ [as can be inferred from the fact that the characteristic bandwidths defined in Eq. (20) go to infinity as $l_{c} \rightarrow 0$ ]. The correlation functions (A10a) and (A10b) are then simply the Fourier transforms of sine and cosine hyperbolic functions of $\sigma A_{0}(\vec{x}, t) l_{c}$, calculated in $\vec{q}-\vec{q}^{\prime}$ and in $\vec{q}+\vec{q}^{\prime}$, respectively. We also verified that these approximate solutions look very similar in shape to those obtained with the complete numerical model (see Fig. 6), although for the chosen crystal length of $4 \mathrm{~mm}$, propagation effects are far from being negligible.

Considering the specific case of a far-field measurement with the $f$ - $f$ lens system described in Sec. V, the Fresnel kernels defined by Eq. (A6) take the form

$$
h_{1}(\vec{x}, \vec{q})=h_{2}(\vec{x}, \vec{q})=-\frac{2 \pi i}{\lambda f} \delta\left(\vec{q}-\frac{2 \pi}{\lambda f} \vec{x}\right)
$$

as can be verified by substituting expression (21b) into Eq. (A7). Equations (A9) reduce then to

$$
\begin{aligned}
\left\langle:\left(\delta N_{1}\right)^{2}:\right\rangle_{\pi^{\prime}}= & \left\langle:\left(\delta N_{2}\right)^{2}:\right\rangle_{\pi^{\prime}} \\
= & \int d \Omega \int d \Omega^{\prime} \int_{Q_{1}} d \vec{q} \int_{Q_{1}} d \vec{q}^{\prime} \mid \int d \vec{q} \int d \vec{q} \\
& \times\left.\left\langle a_{1}^{\text {out }}(\vec{q}, \Omega) a_{1}^{\text {out }}\left(\vec{q}^{\prime}, \Omega^{\prime}\right)\right\rangle\right|^{2}, \\
\left\langle\delta N_{1} \delta N_{2}\right\rangle_{\pi^{\prime}}= & \int d \Omega \int d \Omega^{\prime} \int_{Q_{1}} d \vec{q} \int_{Q_{2}} d \vec{q}^{\prime} \mid \int d \vec{q} \int d \vec{q}^{\prime} \\
& \times\left.\left\langle a_{1}^{\text {out }}(\vec{q}, \Omega) a_{2}^{\text {out }}\left(\vec{q}^{\prime}, \Omega^{\prime}\right)\right\rangle\right|^{2},
\end{aligned}
$$

where $Q_{1}$ and $Q_{2}$ indicate the regions in the spatial frequency plane corresponding to the two symmetrical detection areas $R_{1}$ and $R_{2}$ according to the mapping $\vec{x}$ $\rightarrow 2 \pi / \lambda f \vec{x}$. Knowing that $\left\langle\left(\delta N_{-}\right)^{2}\right\rangle \rightarrow 0$ as $R_{1}, R_{2} \rightarrow \infty$, the localization of the cross- and self-correlation functions (A10a) and (A10b) on a area on the order of $\delta q_{0}^{2}$, for $\vec{q}^{\prime}$ $=\vec{q}$ and $\vec{q}^{\prime}=-\vec{q}$, respectively, guarantees that nearly complete noise reduction is achieved if the area of the two detectors is large compared to the resolution area determined by the pump beam waist, that is, $S_{d i f f}=(\lambda f / 2 \pi)^{2} \delta q_{0}^{2}$.
[1] M.I. Kolobov, Rev. Mod. Phys. 71, 1539 (1999), and references quoted therein.

[2] L.A. Lugiato, M. Brambilla, and A. Gatti, Adv. At., Mol., Opt. Phys. 40, 229 (1999).

[3] C.K. Hong and L. Mandel, Phys. Rev. A 31, 2409 (1985); D.N. Klyshko, Photons and Nonlinear Optics (Gordon and Breach, New York, 1988).

[4] P.H.S. Ribeiro, S. Padua, J.C. Machado da Silva, and G.A. Barbosa, Phys. Rev. A 49, 4176 (1994); D.V. Strekalov, A.V. Sergienko, D.N. Klyshko, and Y.H. Shih, Phys. Rev. Lett. 74, 3600 (1995); T.B. Pittman, Y.H. Shih, D.V. Strekalov, and A.V. Sergienko, Phys. Rev. A 52, R3429 (1995).

[5] D.N. Klyshko, Sov. Phys. JETP 67, 1131 (1988); A.V. Belinskii and D.N. Klyshko, ibid. 78, 259 (1994).

[6] G.A. Barbosa, Phys. Rev. A 54, 4473 (1996).

[7] T.B. Pittman, D.V. Strekalov, D.N. Klyshko, M.H. Rubin, A.V. Sergienko, and Y.H. Shih, Phys. Rev. A 53, 2804 (1996).

[8] A.V. Burlakov, M.V. Chekhova, D.N. Klyshko, S.P. Kulik, A.N. Penin, Y.H. Shih, and D.V. Strekalov, Phys. Rev. A 56, 3214 (1997).

[9] M.H. Rubin, Phys. Rev. A 54, 5349 (1996).

[10] B.E.A. Saleh, A.F. Abouraddy, A.V. Sergienko, and M.C. Teich, Phys. Rev. A 62, 043816 (2000); A.F. Abouraddy, M.B. Nasr, B.E.A. Saleh, A.V. Sergienko, and M.V. Teich, ibid. 63, 063803 (2001); A.F. Abouraddy, B.E.A. Saleh, A.V. Sergienko, and M.C. Teich, J. Opt. Soc. Am. B 19, 1174 (2002).

[11] A. Gatti and L. Lugiato, Phys. Rev. A 52, 1675 (1995); I. Marzoli, A. Gatti, and L.A. Lugiato, Phys. Rev. Lett. 78, 2092 (1997); A. Gatti, H. Wiedemann, L.A. Lugiato, I. Marzoli, G.L. Oppo, and S.M. Barnett, Phys. Rev. A 56, 877 (1997); C. Szwaj, G.-L. Oppo, A. Gatti, and L.A. Lugiato, Eur. Phys. J. D 10, 433 (2000).
[12] A.F. Abouraddy, B.E.A. Saleh, A.V. Sergienko, and M.C. Teich, Opt. Express 9, 498 (2001).

[13] I.V. Sokolov, M.I. Kolobov, A. Gatti, and L.A. Lugiato, Opt. Commun. 193, 175 (2001).

[14] N. Treps, U. Andersen, B. Buchler, P.K. Lam, A. Maitre, H.-A. Bachor, and C. Fabre, Phys. Rev. Lett. 88, 203601 (2002).

[15] L.A. Lugiato, A. Gatti, and E. Brambilla, J. Opt. B: Quantum Semiclassical Opt. 4, S176 (2002).

[16] A. Gatti, E. Brambilla, L.A. Lugiato, and M.I. Kolobov, Phys. Rev. Lett. 83, 1763 (1999).

[17] E. Brambilla, A. Gatti, M. Kolobov, and L.A. Lugiato, Eur. Phys. J. D 15, 117 (2001).

[18] A. Gatti, R. Zambrini, M. San Miguel, and L.A. Lugiato, e-print quant-ph/0306133.

[19] A. Gatti, E. Brambilla, and L.A. Lugiato, Phys. Rev. Lett. 90, 133603 (2003).

[20] Y. Jiang, O. Jedrkiewicz, S. Minardi, P. Di Trapani, A. Mosset, E. Lantz, and F. Devaux, Eur. Phys. J. D 22, 521 (2003).

[21] E. Lantz and F. Devaux, Eur. Phys. J. D 17, 93 (2001).

[22] M.I. Kolobov and I.V. Sokolov, Sov. Phys. JETP 69, 1097 (1989); Phys. Lett. A 140, 101 (1989).

[23] V.G. Dmitriev, G.G. Gurzadyan, and D.N. Nikogosyan, Handbook of Nonlinear Optical Crystals, Springer Series in Optical Sciences (Springer-Verlag, Berlin, 1991); N. Boeuf et al., Opt. Eng. 39, 1016 (2000).

[24] P. Scotto and M. San Miguel, Phys. Rev. A 65, 043811 (2002).

[25] F. Devaux and E. Lantz, Eur. Phys. J. D 8, 117 (2000).

[26] A. Berzanskis, W. Chinaglia, L.A. Lugiato, K.H. Feller, and P. Di Trapani, Phys. Rev. A 60, 1626 (1999).

[27] B.M. Jost, A.V. Sergienko, A.F. Abouraddy, B.E.A. Saleh, and M.C. Teich, Opt. Express 3, 81 (1998). 
[28] M.J. Werner, M.G. Raymer, M. Beck, and P.D. Drummond, Phys. Rev. A 52, 4202 (1995); M.J. Werner and P.D. Drummond, ibid. 56, 1508 (1997).

[29] In the simulations we made use of a reliable Gaussian random number generator which is discussed in R. Toral and A. Chakrabarti, Comput. Phys. Commun. 74, 327 (1993).
[30] See, e.g., W. Press, B. Flannery, S. Teukolsky, and W. Vetterling, Numerical Recipes (Cambridge University Press, Cambridge, 1992).

[31] K. Koch, E.C. Cheung, G.T. Moore, S.H. Chakmakjian, and J.M. Liu, J. Quant. Electr. 31, 769 (1995).

[32] C. Gardiner, Quantum Noise (Springer, Berlin, 1991). 